\title{
Star formation along the Hubble sequence
}

\section{Radial structure of the star formation of CALIFA galaxies}

R. M. González Delgado ${ }^{1}$, R. Cid Fernandes ${ }^{2}$, E. Pérez ${ }^{1}$, R. García-Benito ${ }^{1}$, R. López Fernández ${ }^{1}$, E. A. D. Lacerda ${ }^{1,2}$, C. Cortijo-Ferrero ${ }^{1}$, A. L. de Amorim ${ }^{2}$, N. Vale Asari ${ }^{2}$, S. F. Sánchez ${ }^{3}$, C. J. Walcher ${ }^{4}$, L. Wisotzki ${ }^{4}$, D. Mast ${ }^{5}$, J. Alves $^{6}$, Y. Ascasibar ${ }^{7,8}$, J. Bland-Hawthorn ${ }^{9}$, L. Galbany ${ }^{10,11}$, R. C. Kennicutt Jr. ${ }^{12}$, I. Márquez ${ }^{1}$, J. Masegosa ${ }^{1}$, M. Mollá ${ }^{13}$, P. Sánchez-Blázquez ${ }^{7,8}$, and J. M. Vílchez ${ }^{1}$

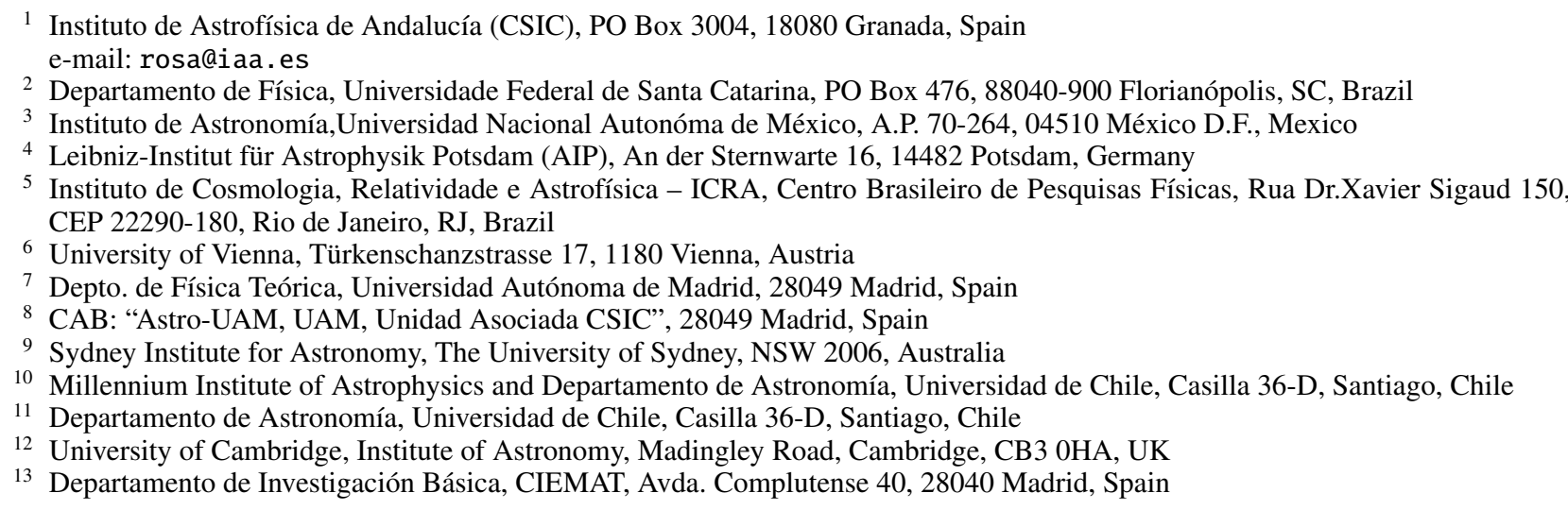

Received 22 January 2016 / Accepted 2 March 2016

\begin{abstract}
The spatially resolved stellar population content of today's galaxies holds important information for understanding the different processes that contribute to the star formation and mass assembly histories of galaxies. The aim of this paper is to characterize the radial structure of the star formation rate (SFR) in galaxies in the nearby Universe as represented by a uniquely rich and diverse data set drawn from the CALIFA survey. The sample under study contains 416 galaxies observed with integral field spectroscopy, covering a wide range of Hubble types and stellar masses ranging from $M_{\star} \sim 10^{9}$ to $7 \times 10^{11} M_{\odot}$. Spectral synthesis techniques are applied to the datacubes to derive 2D maps and radial profiles of the intensity of the star formation rate in the recent past $\left(\Sigma_{\mathrm{SFR}}\right)$, as well as related properties, such as the local specific star formation rate (sSFR), defined as the ratio between $\Sigma_{\mathrm{SFR}}$ and the stellar mass surface density $\left(\mu_{\star}\right)$. To emphasize the behavior of these properties for galaxies that are on and off the main sequence of star formation (MSSF), we stack the individual radial profiles in seven bins of galaxy morphology (E, S0, Sa, Sb, Sbc, Sc, and Sd), and several stellar masses. Our main results are: (a) the intensity of the star formation rate shows declining profiles that exhibit very small differences between spirals with values at $R=1$ half light radius (HLR) within a factor two of $\Sigma_{\mathrm{SFR}} \sim 20 M_{\odot} \mathrm{Gyr}^{-1} \mathrm{pc}^{-2}$. The dispersion in the $\Sigma_{\mathrm{SFR}}(R)$ profiles is significantly smaller in late type spirals (Sbc, Sc, Sd). This confirms that the MSSF is a sequence of galaxies with nearly constant $\Sigma_{\text {SFR }}$. (b) SSFR values scale with Hubble type and increase radially outward with a steeper slope in the inner 1 HLR. This behavior suggests that galaxies are quenched inside-out and that this process is faster in the central, bulge-dominated part than in the disks. (c) As a whole and at all radii, E and S0 are off the MSSF with SFR much smaller than spirals of the same mass. (d) Applying the volume corrections for the CALIFA sample, we obtain a density of star formation in the local Universe of $\rho_{\mathrm{SFR}}=(0.0105 \pm 0.0008) M_{\odot} \mathrm{yr}^{-1} \mathrm{Mpc}^{-3}$, in agreement with independent estimates. Most of the star formation is occurring in the disks of spirals. (e) The volume-averaged birthrate parameter, which measures the current SFR with respect to its lifetime average, $b^{\prime}=0.39 \pm 0.03$, suggests that the present day Universe is forming stars a about one-third of its past average rate. E, S0, and the bulge of early type spirals $(\mathrm{Sa}, \mathrm{Sb}$ ) contribute little to the recent SFR of the Universe, which is dominated by the disks of Sbc, Sc, and Sd spirals. (f) There is a tight relation between $\Sigma_{\mathrm{SFR}}$ and $\mu_{\star}$, defining a local MSSF relation with a logarithmic slope of 0.8 , similar to the global MSSF relation between SFR and $M_{\star}$. This suggests that local processes are important in determining the star formation in disks, probably through a density dependence of the SFR law. The scatter in the local MSSF is driven by morphology-related offsets, with $\Sigma_{\mathrm{SFR}} / \mu_{\star}$ (the local sSFR) increasing from early to late type galaxies, indicating that the shut down of the star formation is more related to global processes, such as the formation of a spheroidal component.
\end{abstract}

Key words. galaxies: evolution - galaxies: stellar content - galaxies: star formation - techniques: spectroscopic

\section{Introduction}

The simple classification scheme introduced by Hubble (1936) is still in use nearly a century later. The reason it remains useful is that the physical properties of galaxies correlate with the morphology in a broad context (e.g., Holmberg 1958; Roberts 1963; Roberts \& Haynes 1994). In particular, the Hubble sequence can 
be described as a sequence in terms of recent star formation, increasing in importance from elliptical (E) to spiral (S) galaxies (e.g., Kennicutt 1983a, 1998).

Contemporary galaxy surveys have mapped this bimodal distribution implicit in the Hubble classification in terms of properties related with their structure (morphology), stellar content, and chemical composition (e.g., Blanton et al. 2003; Baldry et al. 2004; Blanton \& Moustakas 2009; Kauffmann et al. 2003; Mateus et al. 2006; Ascasibar \& Sánchez Almeida 2011; Casado et al. 2015). One population, which is located in the region of the color-magnitude diagram (CMD) known as the red sequence, is composed of galaxies with little star formation, high stellar masses $\left(M_{\star}\right)$, stellar mass surface density $\left(\mu_{\star}\right)$, and light concentration. The other major population, located in the so-called blue cloud in the CMD, consists of galaxies with significant star formation, lower $M_{\star}$ and $\mu_{\star}$, and a low concentration in light. The red sequence is the realm of early type galaxies, whereas galaxies of later Hubble types populate the blue cloud.

Other works have shown that galaxies that populate the blue cloud follow a strong correlation between $M_{\star}$ and the present star formation rate (SFR; Brinchmann et al. 2004; Salim et al. 2007; Renzini \& Peng 2015; Catalán-Torrecilla et al. 2015). The main-sequence of star-forming galaxies (MSSF). The correlation is tight, with only 0.2-0.3 dex dispersion in SFR for a fixed $M_{\star}$ and with a slope that is somewhat smaller than unity, implying that the relative rate at which stars form in galaxies, i.e. the specific star formation rate $S S F R=S F R / M_{\star}$, declines weakly with increasing galaxy mass (Salim et al. 2007; Schiminovich et al. 2007).

Subsequent studies have shown that the MSSF relation persists to at least $z \sim 4$ (e.g., Noeske et al. 2007; Daddi et al. 2007; Elbaz et al. 2007; Peng et al. 2010; Wuyts et al. 2011). These works conclude that most of the star formation in the Universe is produced by galaxies in the main sequence, with starbursts (which deviate upward from the MSSF) contributing only $\sim 10 \%$ to the total star formation rate at $z \sim 2$ (Sanders \& Mirabel 1996; Rodighiero et al. 2011), where the peak of the cosmic star formation rate occurs (e.g., Madau \& Dickinson 2014). A recent study by Speagle et al. (2014) finds that the logarithmic slope of the MSSF relation increases with cosmic time from $\sim 0.6$ at $z \sim 2$ to 0.84 at $z=0$. This implies that the characteristic sSFR of the main sequence population evolves rapidly with redshift (Karim et al. 2011). In fact, Elbaz et al. (2011) show that star formation has decreased by a factor of 20 after $z \sim 2$ and that the corresponding SSFR declined as $t^{-2.2}$, where $t$ is the cosmic epoch.

There is also a substantial population of quenched galaxies that dominate the high end of the mass function, but whose sSFR is significantly lower than in star-forming galaxies (Salim et al. 2007; Schiminovich et al. 2007; Chang et al. 2015). In a simple picture, galaxies evolve along the blue star-forming MSSF, increasing in mass through the accretion of cold gas from the cosmic web and/or through mergers. When it approaches a critical mass, the supply of gas is shut off. Star formation is thus quenched, and the galaxy migrates to the red sequence, where the increase in mass and size may happen through minor mergers (e.g., Faber et al. 2007; Lilly et al. 2013). Although the quenching phase is relevant in the life of a galaxy, it is not clear at which critical mass the galaxy is quenched and whether this is related with a change in the nature of the gas accretion, with heating of the surrounding gas by an AGN or with the formation of a spheroidal component (Martig et al. 2009).
Evidently, this whole field relies on empirical measures of the SFR. There is no shortage of methods of estimating the SFR, each with its virtues and caveats (see Kennicutt \& Evans (2012) for a review). Some gauge the SFR indirectly by quantifying how the radiative output of young stars is reprocessed by gas or dust, as $\mathrm{H} \alpha$ and far infra-red SFR indicators. Direct detection of recently formed stars is best done in the UV, where they outshine older populations by large factors, although dust inevitably introduces uncertainties. Because of the comparable contributions of stellar generations of all ages, the optical continuum is not the cleanest spectral range to work with if one is interested in pinning down the recent star formation history (SFH). It is, nonetheless, the very spectral range where galaxy evolution first started and matured as a research field, as illustrated by the seminal works of Tinsley (1968, 1972), Searle et al. (1973), Gallagher et al. (1984), and Sandage (1986), who first used galaxy optical colors to study how SFHs vary along the Hubble sequence and to predict the cosmic evolution of the SFR. This line of work has been revamped in the past decade or two with the confluence of advances in the spectral modeling of stellar populations (e.g., Leitherer et al. 1999; Bruzual \& Charlot 2003; González Delgado et al. 2005; Maraston 2005; Vazdekis et al. 2010), the development of full spectral synthesis methods (e.g., Panter et al. 2003; Cid Fernandes et al. 2005; Ocvirk et al. 2006; Koleva et al. 2011; Sánchez et al. 2016), and the flood of data from surveys such as the SDSS (Abazajian et al. 2003), which provided abundant observational material to explore these new tools (e.g., Panter et al. 2003, 2008; Heavens et al. 2004; Asari et al. 2007; Tojeiro et al. 2011). A detailed discussion of the uncertainties associated to these methods can be found in the recent reviews by Walcher et al. (2011) and Conroy (2013).

Regardless of the method employed to derive SFHs and SFRs, an important limitation of most studies to date is the lack of spatially resolved information. Galaxies are usually studied as a whole, with observations integrated over their distinct morphological components, or else with data that only partially cover them and are thus prone to aperture effects. Overcoming this limitation requires data of the kind that have only recently started to become available with Integral Field Spectroscopy (IFS) surveys, such as ATLAS3D (Cappellari et al. 2011), CALIFA (Sánchez et al. 2012; Husemann et al. 2013; García-Benito et al. 2015), SAMI (Bryant et al. 2015), and MaNGA (Bundy et al. 2015). These new generation surveys are a step forward to understand the star formation in galaxies and should help us disentangle the contributions of spheroids and disks to the MSSF relation.

Because of its focus on early type galaxies (E, S0, Sa), ATLAS3D essentially avoids starforming systems, so does not constitute an ideal sample to study the MSSF (McDermid et al. 2015). CALIFA, on the other hand, is particularly well suited to this study. First, it includes a large, homogeneous, but diverse sample of galaxies covering the full Hubble sequence, from ellipticals (E0-E7), and lenticulars (S0-S0a), to spirals (Sa to Sd), and a correspondingly wide range of masses $\left(10^{9}\right.$ to $\sim 10^{12} M_{\odot}$, González Delgado et al. 2014b). Second, its large field of view $\left(74^{\prime \prime} \times 64^{\prime \prime}\right.$, with final spatial sampling of $\left.1^{\prime \prime}\right)$ covers the full extent of the galaxies and allows us to spatially map the star formation, as well as to obtain the total integrated SFR. Third, it covers the whole rest-frame optical wavelength at intermediate spectral resolution, which allows us to apply full spectral fits to retrieve the SFHs (and thus recent SFR, too). Finally, the volume-corrected distribution functions of CALIFA are fully compatible with estimates from the full SDSS when accounting 
for large-scale structure (Walcher et al. 2014), which allows the extrapolation of results to the overall cosmic context.

Previous papers in this series used the SFHs of $\sim 100$ 300 galaxies of the CALIFA survey to derive spatially resolved information on the mass growth of galaxies (Pérez et al. 2013), and stellar population properties like the stellar mass surface density, ages, stellar metallicity, and extinction (González Delgado et al. 2014b,a, 2015). We found that massive galaxies grow their stellar mass inside-out, where the signal of downsizing is spatially preserved, with both inner and outer regions growing faster for more massive galaxies. We confirm that more massive galaxies are more compact, older, more metal rich, and less reddened by dust. Additionally, we find that these trends are preserved spatially with the radial distance to the nucleus. Deviations from these relations appear correlated with Hubble type: earlier types are more compact, older, and more metal rich for a given $M_{\star}$, which indicates that quenching is related to morphology.

Here we concentrate on the study of the ongoing star formation of CALIFA galaxies, as derived from full spectral fits of the optical stellar continuum. The goals are 1) to characterize in detail the radial structure of the SFR and SSFR of galaxies in the local Universe; 2) to examine how SFR and sSFR relate to Hubble type; 3 ) to spatially resolve the MSSF relation; and 4) to estimate the contribution of different types of galaxies and their subcomponents to the cosmic star formation rate.

This paper is organized as follows. Section 2 describes the observations and summarizes the properties of the galaxies analyzed here. In Sect. 3 we summarize our method for extracting the SFH and explain how we measure the present SFR. Section 4 presents results on the MSSF relation and how our assumptions affect it. Section 5 deals with the radial structure of the intensity of the star formation rate $\left(\Sigma_{\mathrm{SFR}}\right)$ and related properties such the local specific SFR. We discuss the results and their relation with the cosmic star formation of the local Universe in Sect. 6. Section 7 summarizes our main findings.

\section{Data and sample}

\subsection{Observations and data reduction}

The observations were carried out with the Potsdam MultiAperture Spectrometer PMAS (Roth et al. 2005) in the PPaK mode (Verheijen et al. 2004) at the 3.5m telescope of Calar Alto observatory. PPaK contains 382 fibers of $2.7^{\prime \prime}$ diameter each and a $74^{\prime \prime} \times 64^{\prime \prime}$ field of view (FoV; Kelz et al. 2006). Each galaxy is observed with two spectral settings, V500 and V1200, with spectral resolutions $\sim 6$ (FWHM) and $2.3 \AA$, respectively. The V500 grating covers from 3745 to $7300 \AA$, while the V1200 covers $3650-4840 \AA$. To reduce the effects of vignetting on the data, we combine the observations in the V1200 and V500 setups, calibrated with version 1.5 of the reduction pipeline. We refer to Sánchez et al. (2012), Husemann et al. (2013), and García-Benito et al. (2015) for details on the observational strategy and data processing.

\subsection{Sample: morphological classification}

The CALIFA mother sample consists of 939 galaxies selected from the SDSS survey in the redshift range $z=0.005-0.03$ and with $r$-band angular isophotal diameter of $45-80^{\prime \prime}$. It is primarily a diameter-limited sample to guarantee that the objects fill the $74^{\prime \prime} \times 64^{\prime \prime}$ FoV. It includes a significant number of galaxies

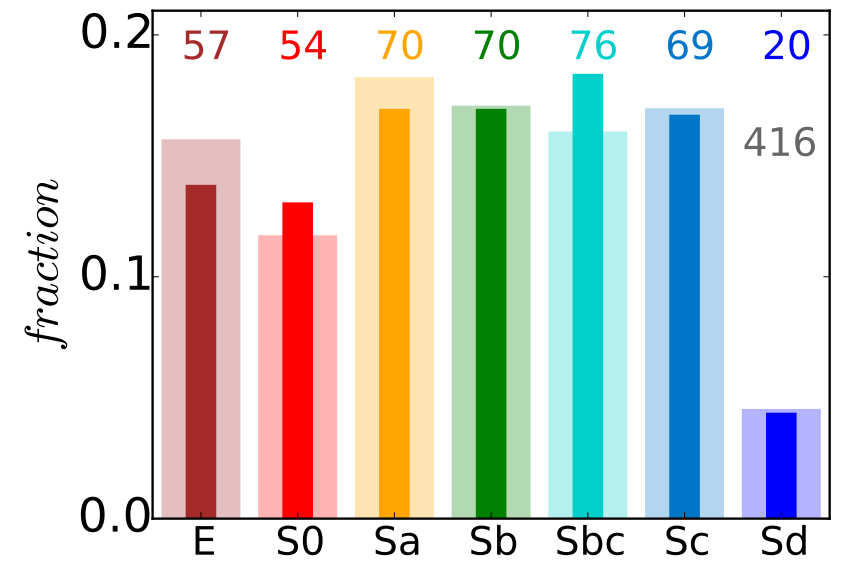

Fig. 1. Comparison of the distribution of Hubble types in the CALIFA mother sample (939 galaxies, bars) and the 416 galaxies analyzed here (filled narrow color bars). The histograms are normalized to unity, so that the two distributions are directly comparable. The number of galaxies in each morphology bin is labeled in color with the same palette used throughout the paper.

in different bins in the CMD and covers a wide and representative range of galaxy types. The galaxies were morphologically classified by five members of the collaboration through visual inspection of the SDSS $r$-band images, averaging the results (after clipping outliers). The sample and its characteristics are fully described in Walcher et al. (2014).

The targets studied in this paper were selected from those observed in both V1200 and V500 setups earlier than January 2015 and excluding type 1 Seyferts and galaxies that show merger or interaction features. This leaves a final sample of 416 galaxies.

As we did in GD15, we group the galaxies into seven morphology bins: E (57 galaxies), S0 (54, including S0 and S0a), $\mathrm{Sa}$ (70, including $\mathrm{Sa}$ and $\mathrm{Sab}), \mathrm{Sb}$ (70), Sbc (76), Sc (69, including Sc and Scd), and Sd (20, including $18 \mathrm{Sd}, 1 \mathrm{Sm}$, and 1 Irr). Figure 1 shows the morphological distribution of our 416 galaxies (filled bars), as well as that of the mother sample (empty bars). Hubble types are labeled with a brown-to-blue (ellipticals to late type spirals) color palette, which is used throughout this paper. The similarity of the two distributions ensures that our subsample is a fair representation of the mother sample. This is an important aspect because it allows us to apply the volume corrections derived by Walcher et al. (2014) to extend the statistical results presented here to those of the galaxy population as a whole.

\section{Stellar population analysis: Mass and star formation rate}

\subsection{Method of analysis}

To extract the stellar population properties from the datacubes we apply the same method as in Pérez et al. (2013), Cid Fernandes et al. (2013, 2014), and González Delgado et al. (2014b,a, 2015). Briefly, after some basic preprocessing steps, such as spatial masking of foreground and background sources, rest-framing, and spectral resampling, the individual spectra that have signal-to-noise ratio $S / N \leq 20$ in a $90 \AA$ window centered at $5635 \AA$ (rest-frame) are coadded into Voronoi zones (Cappellari \& Copin 2003). The resulting 366112 spectra (880 per galaxy, on average) are then fitted with STARLIGHT (Cid Fernandes et al. 2005) using the cluster Grid-CSIC at the 
Table 1. Number of galaxies in each Hubble type and mass interval.

\begin{tabular}{lccccccc}
\hline \hline $\log M_{\star}\left(M_{\odot}\right)$ & $\mathrm{E}$ & $\mathrm{S} 0$ & $\mathrm{Sa}$ & $\mathrm{Sb}$ & $\mathrm{Sbc}$ & $\mathrm{Sc}$ & $\mathrm{Sd}$ \\
\hline$\leq 9.1$ & - & - & - & - & - & 1 & 1 \\
$9.1-9.6$ & - & & - & - & - & 15 & 11 \\
$9.6-10.1$ & - & 1 & 2 & - & 5 & 14 & 5 \\
$10.1-10.6$ & 1 & 3 & 10 & 14 & 18 & 28 & 3 \\
$10.6-10.9$ & 7 & 15 & 10 & 19 & 29 & 4 & 0 \\
$10.9-11.2$ & 16 & 18 & 28 & 22 & 20 & 6 & - \\
$11.2-11.5$ & 19 & 15 & 20 & 14 & 4 & 1 & - \\
$11.5-11.8$ & 13 & 2 & - & 1 & - & - & - \\
$\geq 11.8$ & 1 & - & - & - & - & - & - \\
\hline $\operatorname{total}(416)$ & 57 & 54 & 70 & 70 & 76 & 69 & 20 \\
\hline$\left\langle\log M_{\star}\right\rangle$ & 11.3 & 11.0 & 11.0 & 10.9 & 10.7 & 10.1 & 9.6 \\
$\sigma\left(\log M_{\star}\right)$ & 0.3 & 0.3 & 0.4 & 0.3 & 0.3 & 0.5 & 0.4 \\
\hline
\end{tabular}

Instituto de Astrofísica de Andalucía. The output is then processed through PyCASSO (the Python CALIFA STARLIGHT Synthesis Organizer) to produce a suite of the spatially resolved stellar population properties.

The base used in STARLIGHT's spectral decomposition is a central ingredient in our whole analysis. The results presented here were obtained with base $G M e$, as defined in González Delgado et al. (2014b,a, 2015). This base comprises 235 spectra for simple stellar populations (SSP) drawn from Vazdekis et al. (2010) for populations older than $t=63 \mathrm{Myr}$ and from González Delgado et al. (2005) models for younger ages. The evolutionary tracks are those of Girardi et al. (2000), except for the youngest ages ( 1 and $3 \mathrm{Myr}$ ), which are based on the Geneva tracks (Schaller et al. 1992; Schaerer et al. 1993; Charbonnel et al. 1993). The initial mass function (IMF) is Salpeter. The $Z$ range covers the seven metallicities provided by Vazdekis et al. (2010) models: $\log Z / Z_{\odot}=-2.3,-1.7,-1.3$, $-0.7,-0.4,0$, and +0.22 , but SSPs younger than $63 \mathrm{Myr}$ include only the four largest metallicities. Appendix A presents some comparisons with results obtained with an alternative base built from a preliminary update of the Bruzual \& Charlot (2003) models.

\subsection{Stellar masses}

Our galaxy stellar masses $\left(M_{\star}\right)$ are obtained by adding the masses of each spatial zone (Cid Fernandes et al. 2013; González Delgado et al. 2015). This procedure takes spatial variations of the stellar population properties and stellar extinction into account, something that cannot be done when dealing with integrated light data (i.e., one spectrum per galaxy). Owing to foreground stars or other artifacts, masked spaxels are corrected for in PyCASSO using the stellar mass surface density $\left(\mu_{\star}\right)$ radial profile as explained in González Delgado et al. (2014b).

Both $M_{\star}$ and Hubble type play important roles in this paper, so it is important to know how these two properties relate to each other. Table 1 shows the distribution of galaxies by Hubble type in several bins of $M_{\star}$. The masses range from $8 \times 10^{8}$ to $7 \times 10^{11} M_{\odot}$ (for a Salpeter IMF), and peak at $\sim 10^{11} M_{\odot}$. As expected, $M_{\star}$ correlates with Hubble type (see also González Delgado et al. 2015, particularly their Fig. 2). $\mathrm{E}$ are the most massive galaxies with $\log M_{\star}=11.3 \pm 0.3$ (average \pm dispersion) in solar units, and the least massive galaxies are those in the $\mathrm{Sd}$ bin, with $\log M_{\star}=9.6 \pm 0.4$. The more typical CALIFA galaxy has $\log M_{\star}=10.75$, similar to the Milky Way's mass (Licquia \& Newman 2015).

\subsection{Estimation of the recent SFR from the spectral synthesis}

SFR is usually estimated from $\mathrm{H} \alpha$, far-infrared, or UV luminosities (Kennicutt 1998; Kennicutt \& Evans 2012; Catalán-Torrecilla et al. 2015), which despite their own caveats and limitations, get the job done with conveniently simple, one-line formulae. No such straightforward recipe exists for optical continuum data, however. The reason is that stars of all ages can make comparable contributions to the optical light, and isolating the part due to those formed in the recent past is not a trivial task. It is, however, a feasible one. After all, decomposing a spectrum in terms of stellar populations of different ages is precisely what STARLIGHT does. In fact, an extended version of the code is being developed which incorporates UV, far-infrared, and/or emission line information (López Fernández et al. 2016), all of which should improve its sensitivity to young stars. In any case, as shown by Asari et al. (2007), the standard version of STARLIGHT already performs well in this respect.

This section explains our methodology for computing SFRs. The SFR values themselves are presented in later sections, while the discussion here focuses on how to handle the STARLIGHT output to produce meaningful SFR estimates, what the uncertainties are, and how to improve the results by means of criteria based on ancillary emission line information.

\subsubsection{Choice of a "recent" star formation time scale}

We first specify what we mean by "recent past" by defining $t_{\mathrm{SF}}$ as the age of the oldest stars to be included in the computation of our recent SFR. The mean rate of star formation then follows from a simple summation over all populations younger than $t_{\mathrm{SF}}$ :

$S F R_{x y}=\frac{1}{t_{\mathrm{SF}}} \sum_{t \leq t_{\mathrm{SF}}} M_{\mathrm{txy}}$

where $x y$ denotes a spaxel (or Voronoi zone), and $M_{\mathrm{txy}}$ is the mass initially turned into stars that now have age $t$ at the same $x y$ location. Radial profiles of SFR and galaxy wide rates are trivially obtained by averaging $S F R_{x y}$ over the desired $x y$ region. Similarly, surface densities $\left(\Sigma_{\mathrm{SFR}}\right)$ are obtained by dividing by the corresponding area (a spaxel, a radial ring, the whole galaxy, etc.).

The choice of $t_{\mathrm{SF}}$ is arbitrary, so we sketch some general guidelines to choose a useful value. Naturally, the larger $t_{\mathrm{SF}}$, the more robust the corresponding SFR becomes, since more base elements are summed over in Eq. (1), thus minimizing known degeneracies in stellar population synthesis (e.g., Cid Fernandes et al. 2014). On the other hand, one would like $t_{\mathrm{SF}}$ to be much shorter than the Hubble time (otherwise, SFR and $M_{\star}$ become $\sim$ equivalent quantities). Furthermore, it would be desirable to have a $t_{\mathrm{SF}}$ that resembles the time scale involved in some other independent SFR tracer to which we can compare ours. The natural choice of reference in our case is $\mathrm{H} \alpha$, first because of the widespread use of this tracer, but also because we lack UV or far-infrared data with CALIFA-like spatial resolution for our sample. The $\mathrm{H} \alpha$ luminosity responds to the $h v>13.6 \mathrm{eV}$ radiation field, which is completely dominated by $\lesssim 10$ Myr populations, so we aim at a $t_{\mathrm{SF}}$ of this same order of magnitude.

After some experimentation we chose $t_{\mathrm{SF}}=32 \mathrm{Myr}^{1}$. This choice follows the same rationale (but different data) as in

1 This overly precise looking value of $t_{\mathrm{SF}}$ merely reflects the choice of which ages in our discrete grid to include in the summation in Eq. (1). We chose to include up to the base element at $t=32 \mathrm{Myr}$ (actually $31.62 \mathrm{Myr}$ ). 


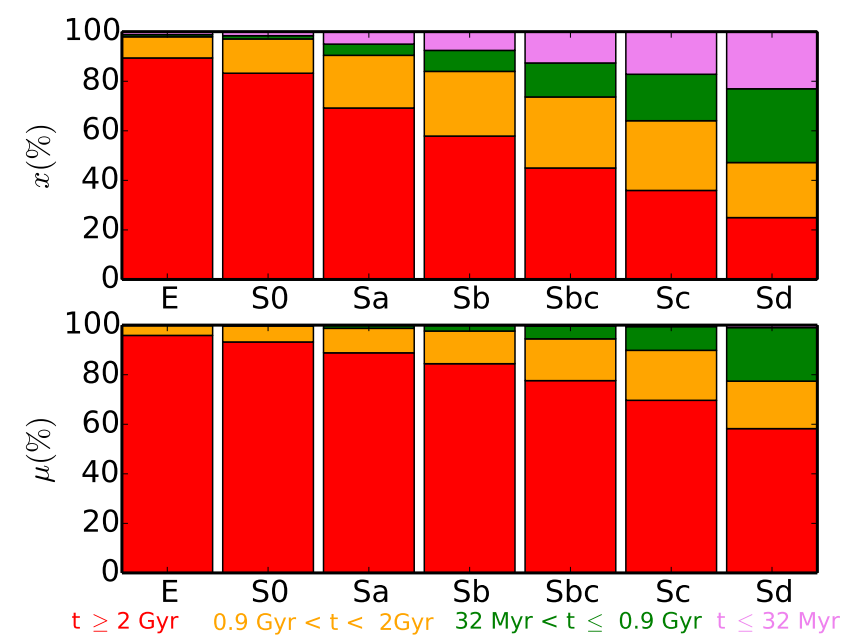

Fig. 2. Average light (upper panel) and mass (bottom) fractions (defined with respect to $\lambda=5635 \AA$, the normalizing wavelength) due to stars in different age ranges as a function of Hubble type. Age ranges are coded by color: the youngest ones, $<32 \mathrm{Myr}$, are in violet (hardly visible in the bottom panel because they carry little mass). Populations from 32 to $900 \mathrm{Myr}$ are shown in green; those from 0.9 to $2 \mathrm{Gyr}$ in orange, and older ones in red.

Asari et al. (2007), who in a study of star-forming galaxies in the SDSS, found that a very similar time scale (25 Myr; see their Fig. 6) produces the best correlation between STARLIGHT and $\mathrm{H} \alpha$-based estimates of SFR. We defer a detailed discussion of this point to a future communication (Lacerda et al., in prep.). For the purposes of this paper, it suffices to say that $t_{\mathrm{SF}}$-values between $\sim 10$ and 100 Myr would lead to the same overall qualitative conclusions.

\subsubsection{SFHs along the Hubble sequence: a condensed view}

Figure 2 tracks the percent contribution in light (top panels) and mass (bottom) of our recent populations $\left(\leq t_{\mathrm{SF}}=32 \mathrm{Myr}\right.$, in magenta) along the Hubble sequence, as well as those of stars in three other intervals: $32 \mathrm{Myr}$ to $0.9 \mathrm{Gyr}$ (green), 0.9 to 2 Gyr (orange), and $\geq 2$ Gyr (red). These four intervals roughly represent populations in which the light is dominated by $\mathrm{O}$, B, A-early F, and later type (lower mass) stars. This strategy of grouping stellar populations in broad age ranges as a way of summarizing SFHs goes back to early studies based on equivalent widths and colors (Bica 1988; Bica et al. 1994; Cid Fernandes et al. 2001), but was also applied in full spectral fitting work (González Delgado et al. 2004; Cid Fernandes et al. 2004, 2005).

The top panels in Fig. 2 show a steady progression of youngand intermediate-age populations along the Hubble sequence. The percent light contribution (at $\lambda=5635 \AA$ ) of populations younger than $32 \mathrm{Myr}$ decreases from $x_{Y}(\%)=23.0$ in Sd galaxies to 17.2 in Sc, 12.6 in Sbc, 5.5 in Sb, and 4.9 in Sa. Because of their low mass-to-light ratio, as well as the tiny time span compared to other bins, these populations are essentially invisible in the bottom panels where the mass fractions are plotted. Indeed, in terms of mass fractions the $<32 \mathrm{Myr}$ populations account for only $0.97,0.59,0.36,0.12$, and $0.05 \%$ for $\mathrm{Sd}, \mathrm{Sc}, \mathrm{Sbc}, \mathrm{Sb}$, and $\mathrm{Sa}$, respectively.

\subsubsection{Star formation in early type galaxies}

The contribution of young stars decreases even more toward the E and S0, but it is not zero with $x_{Y} \sim 2 \%$. Naturally, the reality of populations that account for such little light is questionable. Based on the extensive set of simulations carried out by Cid Fernandes et al. (2014) to evaluate uncertainties in the STARLIGHT results for CALIFA-like data, we estimate the level of noise-induced uncertainty in $x_{Y}$ to be on the order of $3 \%$. Given this, the small $x_{Y}$ fractions identified in E and S0 should be considered noise. However, this $3 \%$ error estimate reflects the level of uncertainty expected for a single spectral fit, whereas the $x_{Y} \sim 2 \%$ in the top left of Fig. 2 reflects an average over 115927 zones inside of the central 3 HLR of $111 \mathrm{E}$ and S0 galaxies. Looking from this statistical angle, one should perhaps take the small $x_{Y}$ fractions in these systems as a sign that they may not be so quiescent after all. There is in fact evidence of some level of star formation in at least some early-type galaxies (Kaviraj et al. 2007). In the context of CALIFA data, Gomes et al. (2016a) have unveiled spiral-arm like features consistent with recent star formation in three early type galaxies.

In any case, at such low $x_{Y}$-levels, one also needs to worry about systematic effects, and the study by Ocvirk (2010) is especially relevant in this respect. He finds that blue horizontal branch stars can easily masquerade as massive young stars in spectral fits, creating the artificial impression of recent star formation in otherwise genuinely old populations. This same effect was in fact detected in previous STARLIGHT-based work on both globular clusters (Cid Fernandes \& González Delgado 2010) and passive galaxies (Cid Fernandes et al. 2011), and ultimately reflects limitations in the modeling of stellar evolution embedded in the SSP models used in our spectral decomposition.

As will soon become clear, the exact values of SFR or $\Sigma_{\mathrm{SFR}}$ in $\mathrm{E}$ and $\mathrm{S} 0$ galaxies are not as important for the purposes of this paper as the fact that their star-forming properties are markedly different from those of later type galaxies, a relative behavior that is safely immune to the uncertainties discussed above.

\subsubsection{The equivalent width of $\mathrm{H} \alpha$ as an ancillary constraint}

The question raised above of the reliability of the recent star formation derived from our optical spectral synthesis analysis is relevant to all our galaxies, and not only to early type ones. We now seek ways to filter out or at least flag objects where STARLIGHT-based SFRs are not reliable enough.

A possible first-cut solution would be to plainly eliminate all data points where $x_{Y}$ is below, say, twice its uncertainty. Adopting the $\sigma\left(x_{Y}\right) \sim 3 \%$ typical uncertainty from the simulations of Cid Fernandes et al. (2014) would then lead to a $x_{Y}>6 \%$ two-sigma criterion to select reliable individual galaxy zones. We note, however, that for statistical reasons, a much less restrictive cut would make more sense for the averaging in Hubble type and radial distance bins performed throughout this paper.

We chose to define a criterion based on entirely different precepts. The idea is to use the $\mathrm{H} \alpha$ emission equivalent width $\left(W_{\mathrm{H} \alpha}\right)$ to guide our decision on whether STARLIGHT-derived SFR is indeed tracing recent star formation reliably or not. The rationale goes as follows: (1) the recent populations we aim to trace are young enough to photoionize the surrounding gas into HII regions, hence produce $\mathrm{H} \alpha$. (2) Stellar evolution plus straightforward nebular physics predicts a minimum value of $W_{\mathrm{H} \alpha}$ in the range of $1-3 \AA$, corresponding to the limit where the interstellar medium is photoionized by hot, old, low mass, evolved stars (HOLMES, as defined by Flores-Fajardo et al. 2011). Systems 

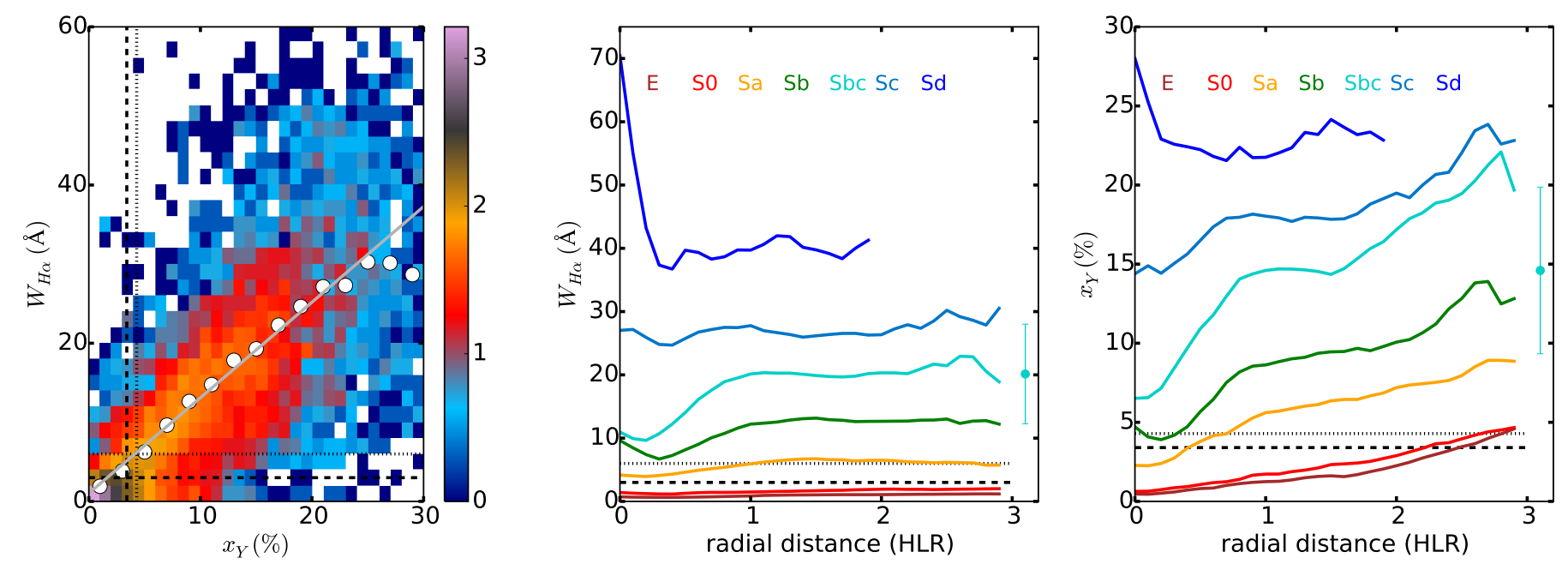

Fig. 3. Left: point density diagram of the equivalent width of the $\mathrm{H} \alpha$ nebular emission, $W_{\mathrm{H} \alpha}$, versus the light fraction due to populations younger than $32 \operatorname{Myr}\left(x_{Y}\right)$ for 12540 radial bins of 416 galaxies. The color bar shows the density of data points on a logarithmic scale. White circles trace the mean relation (obtained from the mean $W_{\mathrm{H} \alpha}$ values in $x_{Y}$-bins). The gray line shows the linear fit to all points: $W_{\mathrm{H} \alpha}(\AA)=1.024+1.208 x_{Y}(\%)$. Horizontal lines are drawn at $W_{\mathrm{H} \alpha}=3$ (dashed) and $6 \AA$ (dotted). Vertical lines mark $x_{Y}=3.4 \%$ (the mean $x_{Y}$ for points with $W_{\mathrm{H} \alpha}<3 \AA$; dashed) and $x_{Y}=4.3 \%$ (the mean $x_{Y}$ for points with $W_{\mathrm{H} \alpha}<6 \AA$; dotted). Middle: mean $W_{\mathrm{H} \alpha}$ radial profiles for galaxies in the seven Hubble type bins. Horizontal lines indicate $W_{\mathrm{H} \alpha}=3$ (dashed), and $6 \AA$ (dotted). Right: radial profile of $x_{Y}$ in the same Hubble type groups. Horizontal lines indicate $x_{Y}=3.4$ (dashed) and $4.3 \%$ (dotted). The dispersion in $W_{\mathrm{H} \alpha}$ (middle panel) and $x_{Y}$ (right panel) measured at 1 HLR in Sb galaxies is shown as an error bar. (The corresponding statistical errors are much smaller.)

with $W_{\mathrm{H} \alpha} \lesssim 3 \AA$ must therefore have stopped forming stars very long ago, a regime dubbed as "retired galaxy" by Stasińska et al. (2008) and Cid Fernandes et al. (2011).

This whole scheme is based on the idea that both $x_{Y}$ and $W_{\mathrm{H} \alpha}$ trace recent star formation, a corollary of which is that they are correlated. This expectation is fully born out by our data, as seen in the lefthand panel of Fig. 3. The plot shows a (log-scale) density map of $W_{\mathrm{H} \alpha}$ versus $x_{Y}$ for 11894 radial points where $\mathrm{H} \alpha$ emission could be measured, along with the mean $W_{\mathrm{H} \alpha}$ values in $x_{Y}$ bins and the corresponding linear fit. We point out that, although expected, this empirical correlation is in no sense tautological, since the two axes are derived from completely independent observables ${ }^{2}$. In fact, we regard this independence as an added benefit of our approach.

Points with $W_{\mathrm{H} \alpha}<3 \AA$ in the lefthand panel of Fig. 3 have on average $\bar{x}_{Y}=3.4 \%$. This limit on $W_{\mathrm{H} \alpha}$ is based both on the observed bimodal distribution of $W_{\mathrm{H} \alpha}$ in local Universe galaxies and on long-known theoretical expectations (Cid Fernandes et al. 2011) ${ }^{3}$. Sánchez et al. (2015) propose a more stringent $W_{\mathrm{H} \alpha}>6 \AA$ cut to isolate regions ionized by young stars. The mean $x_{Y}$ for populations with weaker $W_{\mathrm{H} \alpha}$ is $4.3 \%$, very close to that obtained with the Cid Fernandes et al. (2011) criterion. It is clear that adopting either of the cuts $\left(W_{\mathrm{H} \alpha}, x_{Y}\right)=(3 \AA, 3.4 \%)$ or $(6 \AA, 4.3 \%)$ (see Fig. 3 ) should not lead to significantly different results.

Before elaborating more on the effects of $W_{\mathrm{H} \alpha}$ and $x_{Y}$-based reliability criteria, we first examine how these two properties vary across the face of galaxies.

\footnotetext{
2 Strictly speaking $W_{\mathrm{H} \alpha}$ does depend on the STARLIGHT run, since the line flux is measured over the residual spectrum obtained after subtracting the STARLIGHT fit, but this is only a second-order dependence.

3 The $W_{\mathrm{H} \alpha}$ values expected for galaxies where HOLMES dominate the ionizing flux is in the 0.5-2.4 $\AA$ range (Binette et al. 1994; Cid Fernandes et al. 2011; Gomes et al. 2015). Our $W_{\mathrm{H} \alpha}<3 \AA$ limit adds a (small) safety cushion to this prediction.
}

\subsubsection{The radial profiles or $W_{\mathrm{H} \alpha}$ and $x_{Y}$}

The middle and righthand panels in Fig. 3 show the average radial profiles of $W_{\mathrm{H} \alpha}$ and $x_{Y}$ for the seven morphological bins. As in our previous papers (e.g., González Delgado et al. 2014b), these average profiles are constructed by first expressing the radial distance for each galaxy in units of the corresponding half light radius (HLR), defined as the length of the elliptical aperture along the major axis that contains half of the total flux at $5635 \AA$ (rest frame) within the field of view of PPaK.

The vertical ordering of Hubble types in the middle and righthand panels of Fig. 3 follows the expected tendency, with late-type systems being more star-forming than early type ones. Focusing on the lower part of the plots, we see that E and SO have mean $W_{\mathrm{H} \alpha}<3 \AA$ at all locations, confirming that the extended $\mathrm{H} \alpha$ emission in these systems is consistent with being produced from photoionization by old stars (Sarzi et al. 2006; Kehrig et al. 2012; Papaderos et al. 2013; Singh et al. 2013; Gomes et al. 2015). Whatever little star formation remains in these early type galaxies, it is the exception, not the rule. Furthermore, such residual star formation would be located toward the outskirts of these galaxies, as indicated by the rise in their $x_{Y}(R)$ profiles, reaching 3-5\% for $R \gtrsim 2$ HLR).

Moving to Sa galaxies, we see that, on average, they have $W_{\mathrm{H} \alpha}>3 \AA$ at all radii, although they get close to this limit in their central regions (probably reflecting a contribution from retired bulges). Also, except for the central 0.5 HLR, $x_{Y}(R)$ values are all above the $3.4 \%$ line. Beyond 1 HLR their mean $W_{\mathrm{H} \alpha}$ oscillates around $6 \AA$, so a $<6 \AA$ cut would remove significant portions of their disks. Finally, Fig. 3 shows that whichever reliability cut we chose to apply would make little difference for $\mathrm{Sb}$ and later type galaxies.

In what follows we give more emphasis to results obtained by applying a $x_{Y}>3.4 \%$ cut when computing SFR through Eq. (1), but results obtained with the alternative $W_{\mathrm{H} \alpha}>3$ or $6 \AA$ criteria are also presented for completeness. In the next 

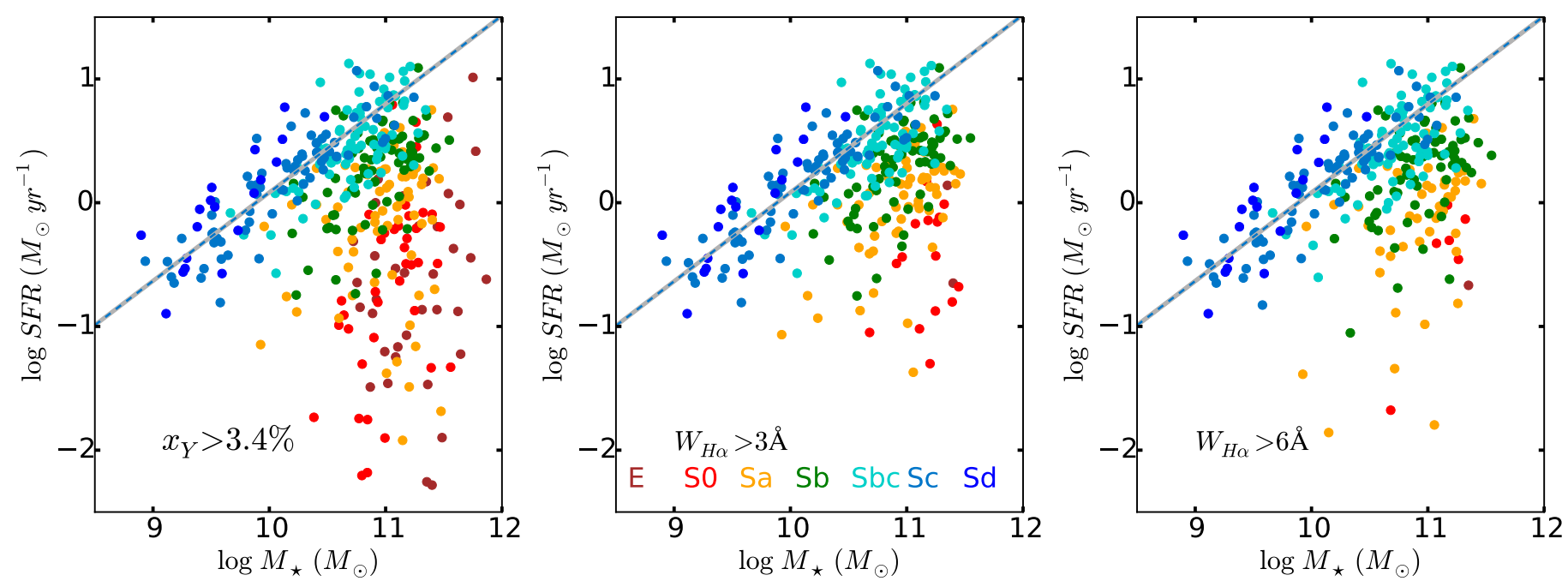

Fig. 4. Relation between SFR and stellar mass for 416 CALIFA galaxies, color coded by Hubble type. A linear fit to the points of Sc galaxies is shown as a gray-blue dashed line. Different panels show the results obtained considering different selection criteria imposed upon the individual $x y$ spaxels included in the computation of the galaxy's total $S F R=\sum_{x y} S F R_{x y}$ : Only $x_{Y}>3.4 \%$ (left), $W_{\mathrm{H} \alpha}>3 \AA$ (middle), and $W_{\mathrm{H} \alpha}>6 \AA$ (right).

section we explore the impact of these three different criteria on the galaxy-wide SFR and the correlation between $M_{\star}$ and SFR.

\section{The global main sequence of star-forming galaxies}

As reviewed in the introduction, the main sequence of starforming galaxies (MSSF) is the name given to the correlation between SFR and $M_{\star}$ (Noeske et al. 2007). This correlation has been found in star-forming galaxies of the local Universe (Brinchmann et al. 2004) and seen to persist at least to redshift ４ (Peng et al. 2010; Wuyts et al. 2011). The logarithmic slope of the relation varies in the range from 0.4 to 1 , depending on the galaxy selection criteria and on the indicator used to estimate the SFR (Speagle et al. 2014). Recently, Renzini \& Peng (2015) have proposed to characterize the main sequence by the ridge line of the star-forming peak in a 3D SFR- $M_{\star}$-number plot obtained with the SDSS sample. Their objective definition leads to a best fit line given by $\log \operatorname{SFR}\left(M_{\odot} \mathrm{yr}^{-1}\right)=(0.76 \pm 0.01) \times$ $\log M_{\star}\left(M_{\odot}\right)-(7.64 \pm 0.02)$.

Figure 4 shows three versions of the $\log$ SFR vs. $\log M_{\star}$ relation obtained with our data and methods. We call this relation the "global MSSF", in contrast to the "local MSSF" where SFR and $M_{\star}$ values are replaced by their respective surface densities (cf. Sect. 6.3 below and Cano-Díaz et al. 2016). The total SFR is calculated for each galaxy using Eq. (1) and adding the contribution of all spaxels that verify $x_{Y}>3.4 \%$ (panel a), $W_{\mathrm{H} \alpha}>3 \AA$ (b), or $W_{\mathrm{H} \alpha}>6 \AA$ (c). Galaxies are color-coded according to their morphology.

The dashed gray-blue lines in all panels show $\log S F R=$ $a \log M_{\star}+b$ fits obtained for Sc galaxies. The correlation is very similar in the three panels with a logarithmic slope $a=0.77$ and zero point of $b=-7.66$. These values are indistinguishable from those obtained by Renzini \& Peng (2015) for the whole SDSS sample. This coincidence is not surprising because Sc and Sbc are the galaxies that contribute the most to the local star formation rate density (Sect. 6.1), which are the ones that produce the ridge line in the MSSF relation.

As is clear from Fig. 4 , the spread in SFR at fixed $M_{\star}$ is related to galaxy morphology. Table 2 lists the slopes and zero points obtained for subsamples of fixed Hubble type. The slopes
Table 2. Parameters of $\log S F R\left(M_{\odot} \mathrm{yr}^{-1}\right)=a \log M_{\star}\left(M_{\odot}\right)+b$ fits of the global MSSF for galaxies of different morphologies.

\begin{tabular}{lccccc}
\hline \hline Morph. & $\mathrm{Sa}$ & $\mathrm{Sb}$ & $\mathrm{Sbc}$ & $\mathrm{Sc}$ & $\mathrm{Sd}$ \\
\hline logarithmic slope $(a)$ & 0.34 & 0.65 & 0.71 & 0.77 & 0.94 \\
zero-point $(b)$ & -3.87 & -6.83 & -7.05 & -7.66 & -9.12 \\
$\log \operatorname{SFR}\left(M_{\star}=10^{10} M_{\odot}\right)$ & -0.47 & -0.33 & -0.05 & -0.04 & 0.28 \\
\hline
\end{tabular}

Notes. They are obtained for panel (a) in Fig. 4. For convenience, the corresponding SFR for a $10^{10} M_{\odot}$ galaxy is also listed (in $M_{\odot} \mathrm{yr}^{-1}$ ). The slope is significantly shallower in Sa than in later spirals because most of the Sa galaxies are off the MSSF.

steepen systematically from 0.34 for $\mathrm{Sa}$ to 0.94 for $\mathrm{Sd}$ galaxies. This range is essentially the same as the $0.4-1$ quote by Speagle et al. (2014) since resulting from different selection criteria. The flattening for the early types also explains why many works obtain a flattening of the MSSF relation at increasing $M_{\star}$ (e.g., Brinchmann et al. 2004; Peng et al. 2010). It is clear in Fig. 4 that the bending in the main sequence, at least in our sample, is produced by the inclusion of large bulges, such as those in $\mathrm{Sa}$ and $\mathrm{S} 0$, and also E, where the star formation is already quenched or in the process of being quenched. These galaxies (Sa, SO, and E) are the most massive ones in our sample, but they contribute little to the cosmic star formation (as seen in Sect. 6.1), because they are clearly off below the MSSF.

Figure 4 shows that the three alternative cuts defined in the previous section produce practically identical MSSF when galaxies later than $\mathrm{Sa}$ are considered. The differences in SFR between the panels become significant in the high $M_{\star}$ and low SFR regime typical of early type galaxies. The masses are the same from panel to panel, since all spaxels contribute to $M_{\star}$. What changes is the list of spaxels entering the computation of SFR of each galaxy, hence the differences in the total rate. In practice we obtain a more extended quenched cloud in the left panel than in middle and right panels. This happens because the $W_{\mathrm{H} \alpha}$-based cuts eliminate most $\mathrm{E}$ and several $\mathrm{S} 0$ and $\mathrm{Sa}$ galaxies altogether, while $x_{Y}>3.4 \%$ is not as restrictive. This again suggests that our estimation of the SFR in E and S0 is uncertain, and our method only provides an upper limit to the real SFR. 

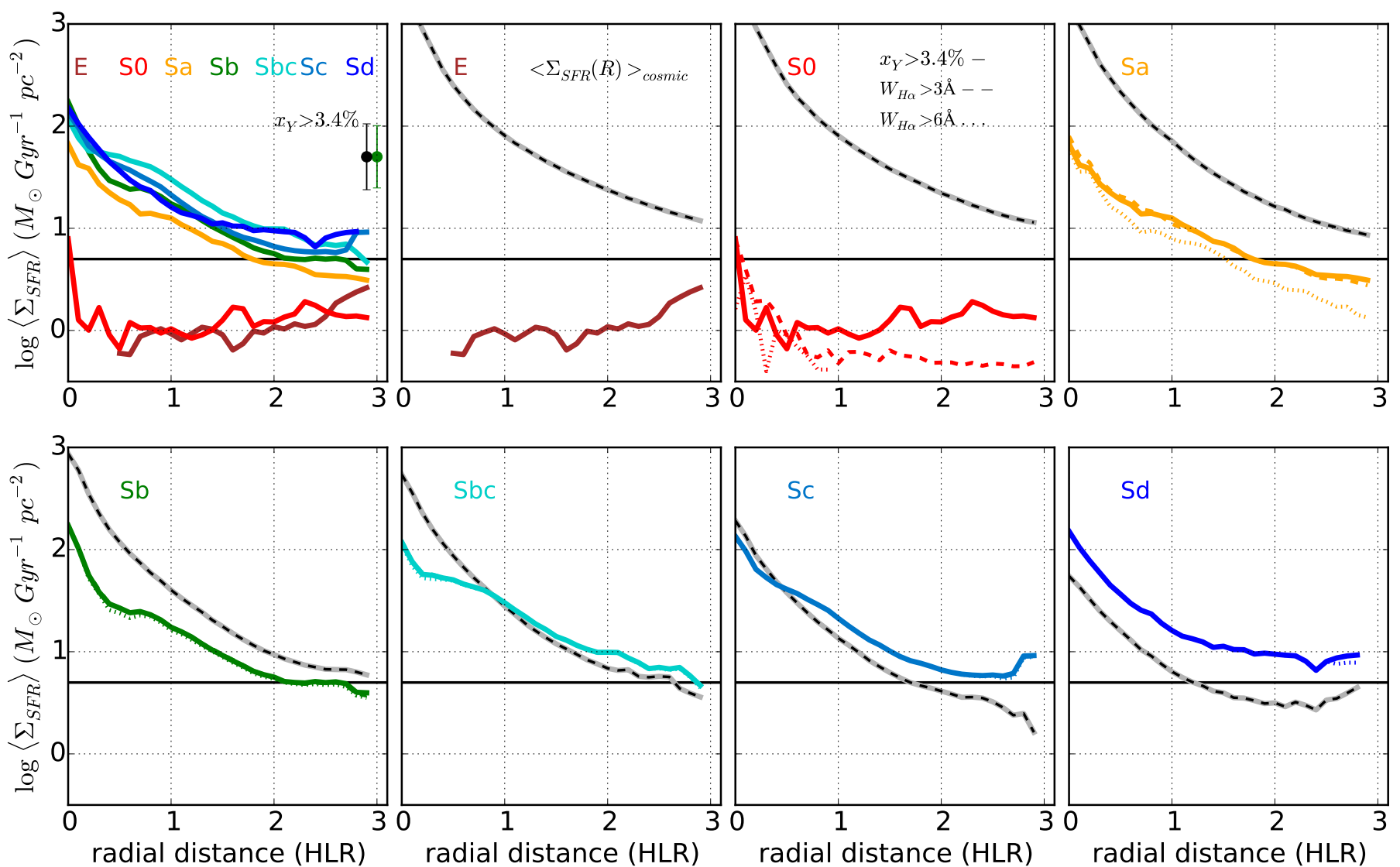

Fig. 5. Upper left: radial profiles (in units of HLR) of the star formation rate surface density $\left(\Sigma_{\mathrm{SFR}}\right)$, averaged in seven morphology bins. The horizontal line at $5 M_{\odot} \mathrm{Gyr}^{-1} \mathrm{pc}^{-2}$ indicates the average $\Sigma_{\mathrm{SFR}}$ in the Milky Way (MW). Only the locations where the light fraction due to young populations is higher than $3.4 \%$ are included. Error bars show the dispersion (not the uncertainty) in $\log \Sigma_{\mathrm{SFR}}$ for all the spirals (black) and for Sb galaxies (green). Other panels: for each Hubble type, solid, dashed, and dotted lines show the mean profiles obtained by excluding $x_{Y}<3.4 \%$, $W_{\mathrm{H} \alpha}<3 \AA$, and $W_{\mathrm{H} \alpha}<6 \AA$, respectively. The gray-dashed lines show $\left\langle\Sigma_{\mathrm{SFR}}(R)\right\rangle_{\text {cosmic }}$ profiles, the SFR surface density profiles obtained assuming a constant rate of star formation throughout the Hubble time.

\section{Radial structure of the recent star formation}

We now present a series of results related to spatially resolved SFR measurements of CALIFA galaxies. We focus on the radial structure of the star formation rate surface density, $\Sigma_{\mathrm{SFR}}$ (also referred to as the intensity of star formation), and the local specific star formation rate, $\Sigma_{\mathrm{SFR}} / \mu_{\star}$.

Using PyCASso we obtain, for each galaxy, 2D maps of the recent SFR computed as in Eq. (1) with $t_{\mathrm{SF}}=32 \mathrm{Myr}$. Each 2D map is then azimuthally averaged to obtain the radial variation of the $\Sigma_{\mathrm{SFR}}$. Only spaxels that meet the criterion of $x_{Y}>3.4 \%$ are included in the azimuthal average. Elliptical apertures $\Delta R=0.1 \mathrm{HLR}$ in width are used to extract the radial profiles, with ellipticity and position angle obtained from the moments of the $5635 \AA$ flux image. We express the radial distance in units of HLR to allow comparison of the profiles of individual galaxies and to produce stacks as a function of Hubble type and/or stellar mass.

\subsection{Radial profiles of $\Sigma_{\mathrm{SFR}}$ and the role of morphology}

Figure 5 shows azimuthally averaged radial profiles of $\Sigma_{\mathrm{SFR}}$ stacked by Hubble type. The upper lefthand panel shows the results for all the seven morphological classes together.

All spirals show $\Sigma_{\mathrm{SFR}}(R)$ decreasing with radial distance, with a typical gradient (measured in the central 1 HLR) $\Delta \log \Sigma_{\mathrm{SFR}}=-0.78 \mathrm{dex} / \mathrm{HLR}$. Interestingly, the $\Sigma_{\mathrm{SFR}}(R)$ at any radius falls within a relatively tight range of values. At $R=$ 1 HLR our average $\Sigma_{\mathrm{SFR}}$ is $20 M_{\odot} \mathrm{Gyr}^{-1} \mathrm{pc}^{-2}$ with a dispersion of 0.13 dex between spirals of different Hubble types. This is about one to two orders of magnitude less than the global $\Sigma_{\text {SFR }}$ measured in starbursts and local Lyman break analogs (Heckman et al. 2005), but consistent with the value obtained by Schiminovich et al. (2007) for a complete sample of GALEX star-forming galaxies ${ }^{4}$.

The plot also illustrates how E and S0 are clearly distinct from the spirals. Their radial profiles are flat (except for some slight increase at the center of S0). The $\Sigma_{\mathrm{SFR}}$ at $1 \mathrm{HLR}$ is $\sim 1 M_{\odot} \mathrm{Gyr}^{-1} \mathrm{pc}^{-2}$, a 20 -fold decrease from spirals (maybe more given that our estimates of SFR for early types are probably upper limits).

Each of the other panels in Fig. 5 shows the radial profile of $\Sigma_{\text {SFR }}$ for each Hubble type, now computed with each of our three reliability cuts. As already discussed, imposing $x_{Y}>3.4 \%$ (solid line), $W_{\mathrm{H} \alpha}>3$ (dashed), or $6 \AA$ (dotted) makes no difference for galaxies later than $\mathrm{Sb}$, so much so that the three $\Sigma_{\text {SFR }}$ profiles are hardly distinguishable. The effects of the somewhat more restrictive $W_{\mathrm{H} \alpha}$-cuts start to be noticed in Sa galaxies, become evident in S0 (factor of $\sim 2$ difference), and grow even larger in $\mathrm{E}$ (where the dotted and dashed lines fall off below the plot limits). As previously discussed, though some of our E galaxies

4 They divide half of the total SFR (derived from the UV luminosity) by an area equal to $\pi \mathrm{HLR}^{2}$. 

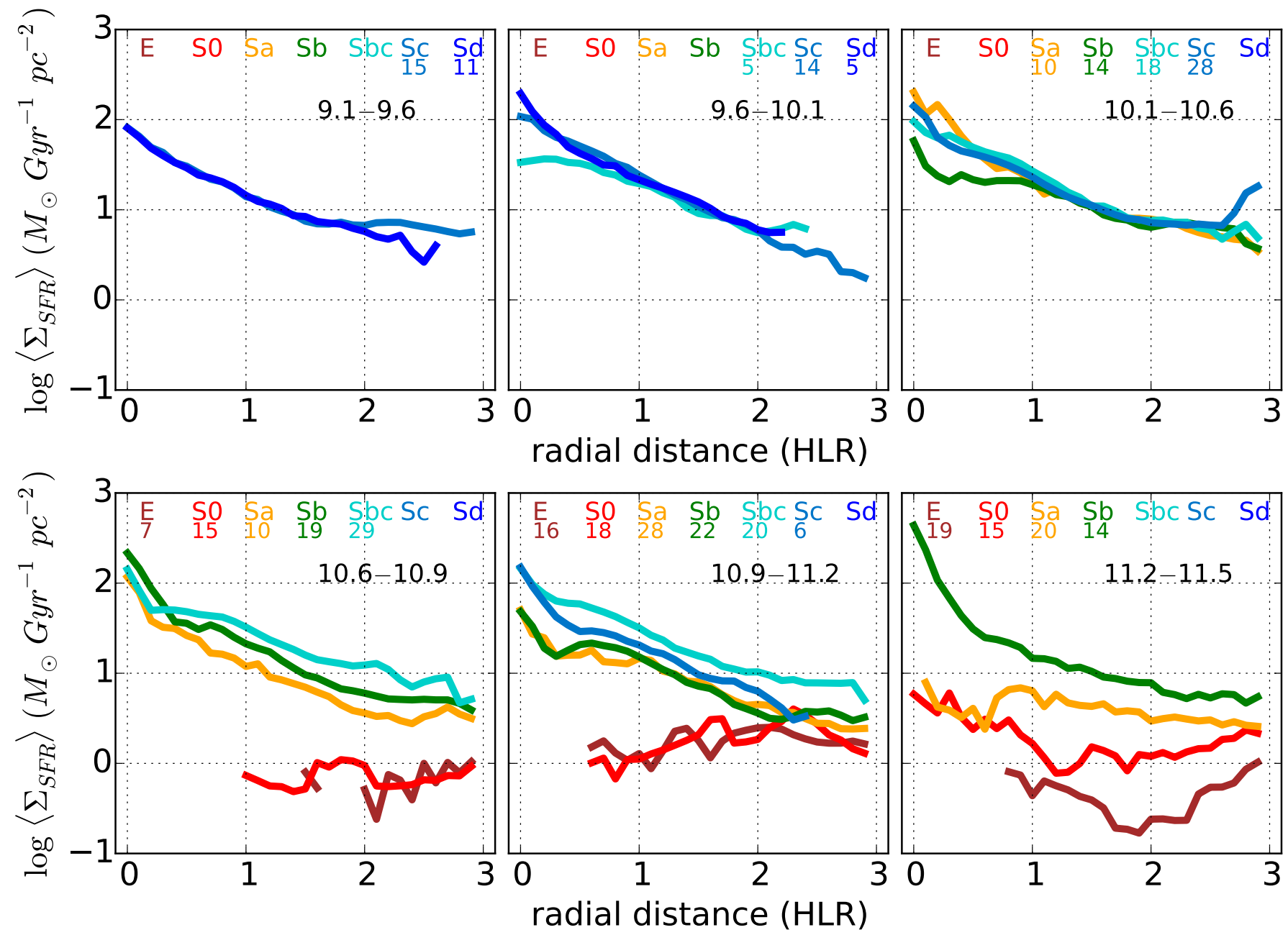

Fig. 6. Radial profiles of $\Sigma_{\mathrm{SFR}}$ for different Hubble types in six galaxy stellar mass bins. From top left to bottom right: $\log M_{\star}\left(M_{\odot}\right)=9.1-9.6$, 9.6-10.1, 10.1-10.6, 10.6-10.9, 10.9-11.2, 11.2-11.5. In each panel, the average profile for each Hubble type is plotted if more than five galaxies have masses in the corresponding $M_{\star}$-bin. The number of galaxies in each bin is also labeled and color-coded.

do exhibit signs of recent star formation (Gomes et al. 2016b), most can be regarded as quenched systems, which retired from forming stars long ago. We note in passing that although our estimates for $\mathrm{E}$ and $\mathrm{S} 0$ are very uncertain, the typical value of $\sim 1 M_{\odot} \mathrm{Gyr}^{-1} \mathrm{pc}^{-2}$ is consistent with the global $\Sigma_{\mathrm{SFR}}$ in earlytype galaxies estimated by Schiminovich et al. (2007).

The individual panels of Fig. 5 present two other lines that allow for interesting comparisons. The first is the horizontal line at $5 M_{\odot} \mathrm{Gyr}^{-1} \mathrm{pc}^{-2}$, which indicates the $\Sigma_{\mathrm{SFR}}$ of the Milky Way. This value is obtained by dividing the recent SFR of the Milky Way (MW), 1.6 $M_{\odot} \mathrm{yr}^{-1}$, by the area of a disk of radius $1.2 \times$ the Galactocentric radius of the Sun, $8.33 \mathrm{kpc}$ (Licquia \& Newman 2015). This distance is equivalent to 2 HLR of typical Sb-Sbc CALIFA galaxies. The results in Fig. 5 suggest that, in the inner $\sim 2$ HLR, most spirals in the main sequence have $\Sigma_{\mathrm{SFR}}$ higher than the average $\Sigma_{\text {SFR }}$ in the Milky Way. This is expected because it is known that the SFR in the MW is significantly lower than in other spirals of similar type and mass (see Kennicutt \& Evans 2012, particularly the discussion around their Fig. 7, where the $\Sigma_{\text {SFR }}$ profiles of the Galaxy and NGC 6946 are compared).

The dashed gray-black lines in Fig. 5 present another useful reference to compare our results to. They represent the $\Sigma_{\mathrm{SFR}}(R)$ the galaxy should have if it formed stars at a constant rate throughout the lifetime of the Universe. Except for the correction for returned mass (a simple scaling factor under most circumstances), these $\left\langle\Sigma_{\mathrm{SFR}}(R)\right\rangle_{\text {cosmic }}$ profiles reflect the stellar mass surface density profile divided by $14 \mathrm{Gyr}$.

$\mathrm{E}$ and S0 are clearly quenched, with $\Sigma_{\mathrm{SFR}} \sim 2$ dex below their $\left\langle\Sigma_{\mathrm{SFR}}(R)\right\rangle_{\text {cosmic }}$. Sa and Sb galaxies, although still active in forming stars, do so at a lower rate than in the past. In contrast, spirals of later types are forming stars at a rate similar to or higher than their $\left\langle\Sigma_{\mathrm{SFR}}(R)\right\rangle_{\text {cosmic }}$. In the central 0.5 HLR of Sbc, presumably their bulges, $\Sigma_{\mathrm{SFR}}$ have decreased with respect to the past, although the disk (outside 1 HLR) is currently forming stars somewhat more actively than in the past. The intersection of the $\Sigma_{\mathrm{SFR}}$ and $\left\langle\Sigma_{\mathrm{SFR}}(R)\right\rangle_{\text {cosmic }}$ curves occurs even closer to the nucleus in Sc, while in Sd the current intensity of star formation exceeds the past average at all radii.

\subsection{The dependence of $\Sigma_{\mathrm{SFR}}(R)$ on stellar mass}

As usual, it is difficult to disentangle the relative roles of morphology and $M_{\star}$, but CALIFA has grown large enough a sample to attempt to tackle this issue by plain brute force statistics. Figure 6 shows $\Sigma_{\mathrm{SFR}}(R)$ profiles as a function of both $M_{\star}$ and morphology. Besides the seven Hubble types we now break up the sample in six mass bins: $\log M_{\star}\left(M_{\odot}\right)=11.5-11.2$, 11.2-10.9, 10.9-10.6, 10.6-10.1, 10.1-9.6, and 9.6-9.1. In each panel (one per $M_{\star}$ bin), the average profile for each Hubble type is plotted if it contains more than five galaxies. These plots allow 
us to evaluate how $\Sigma_{\mathrm{SFR}}(R)$ changes with Hubble type for galaxies of similar mass.

An inspection of Fig. 6 shows that spirals with $M_{\star} \lesssim 4 \times$ $10^{10} M_{\odot}$ (top panels) have very similar $\Sigma_{\text {SFR }}$ profiles. When relevant, the differences occur in the inner regions. Above this mass, the profiles start to disperse, although they are still packed in a relatively narrow range of $\Sigma_{\mathrm{SFR}}$ values. This high degree of uniformity is a remarkable result when taking into consideration that the sample covers all types of spirals and two orders of magnitude in galaxy mass. In Sect. 6.4 we speculate that this behavior is intimately linked to the tightness of the MSSF.

In contrast, $\mathrm{E}$ and $\mathrm{S} 0$ have $\Sigma_{\mathrm{SFR}}$ profiles well below those in spirals of similar mass. This suggests that in massive galaxies with a large spheroidal component, the star formation is significantly quenched in the whole galaxy. However, this effect seems to be more relevant in the centers than in the outskirts, as suggested by the flat profiles in E and S0 in comparison with the radially decreasing $\Sigma_{\mathrm{SFR}}(R)$ profile in spirals. The most massive Sa galaxies in the sample show bimodal behavior with a smooth decrease in $\Sigma_{\mathrm{SFR}}(R)$ outwards of 1 HLR and a relative rate in the central part that is almost flat and significantly depressed with respect to spirals of later types. Again, this points to the relevance that the formation of a big bulge may have in quenching the star formation in galaxies.

\subsection{Radial structure of the local specific star formation rate}

For a galaxy, the specific star formation rate is defined by $S S F R=$ $S F R / M_{\star}$. Overlooking trivial multiplicative factors (see Eq. (2)), it gives a measure of the relative rate at which stars are now forming in a galaxy with respect to the past average rate. Because the relation between SFR and $M_{\star}$ is sublinear (e.g., Fig. 4), the sSFR declines with galaxy mass. Also, because of the tightness of the MSSF relation, star-forming galaxies occupy a correspondingly tight locus in the sSFR vs. $M_{\star}$ space, but bulge-dominated galaxies display a much wider spread of sSFR at a fixed galaxy mass (Schiminovich et al. 2007; Salim et al. 2007 - see also Fig. 4).

In analogy with the global sSFR, CALIFA data allow us the study of the local sSFR, defined by the ratio $\Sigma_{\mathrm{SFR}} / \mu_{\star}$, which measures the relative rate of ongoing star formation with respect to the past in each position in a galaxy. Figure 7 shows the results of stacking the $\operatorname{SSFR}(R)=\Sigma_{\mathrm{SFR}}(R) / \mu_{\star}(R)$ profiles by Hubble type. These profiles show a clear ranking with morphology, increasing from early to late Hubble type. We obtain, at $R=1$ HLR, $\log S S F R\left(\mathrm{Gyr}^{-1}\right)=-2.94,-2.85,-1.68,-1.34,-0.95$, -0.77 , and -0.59 for E, S0, Sa, Sb, Sbc, Sc, and Sd bins, respectively. This ordering is preserved at any given radial distance, as is also the case with other stellar population properties, such as mean stellar age, metallicity, and $\mu_{\star}$ (González Delgado et al. 2015).

Figure 5 shows that $\Sigma_{\mathrm{SFR}}(R)$ profiles are very similar for all spirals, so the scaling of $\operatorname{sSFR}(R)$ seen in Fig. 7 is a direct consequence of the variation in $\mu_{\star}(R)$ with Hubble type, increasing from $\mu_{\star}(R=1 \mathrm{HLR}) \sim 100$ to $1000 M_{\odot} \mathrm{pc}^{-2}$, from $\mathrm{Sd}$ to $\mathrm{Sa}$ galaxies. The opposite happens for early-type galaxies, with the $\operatorname{sSFR}(R)$ profiles of $\mathrm{E}$ and $\mathrm{S} 0$ galaxies running well below those of $\mathrm{Sa}$, while their $\mu_{\star}(R)$ profiles are similar (González Delgado et al. 2015). The difference in this case comes from the much lower levels of star formation in these systems.

All the galaxies have outwardly increasing sSFR profiles. Figure 7 shows that in spirals, $\operatorname{sSFR}(R)$ grows faster with radius in the inner 1 HLR than outward, probably signaling the bulgedisk transition. Assuming that the central 0.1 HLR is dominated

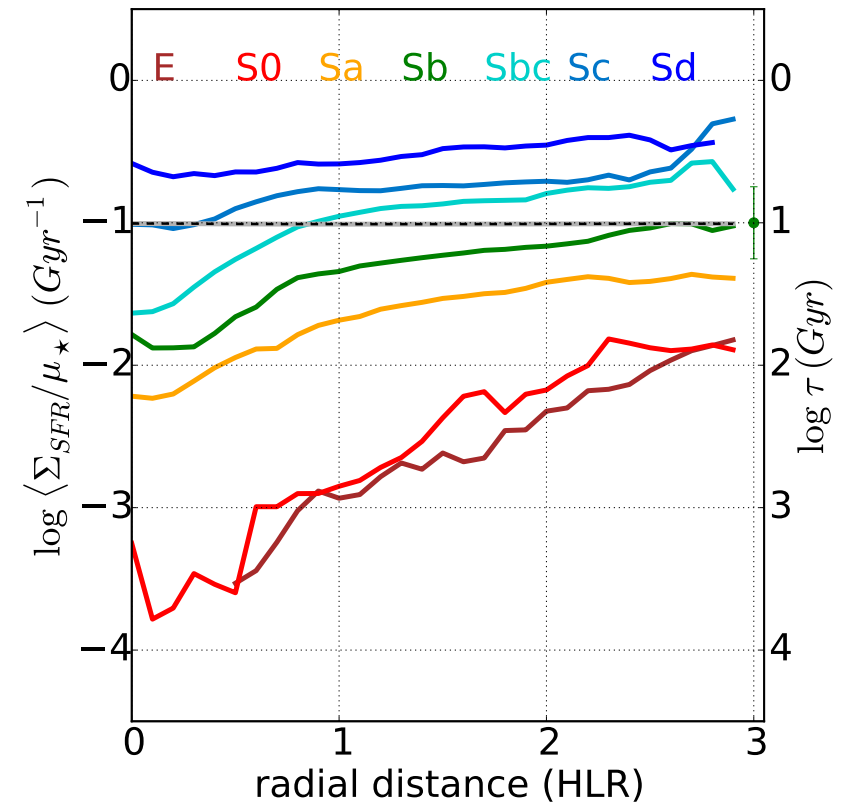

Fig. 7. As for the upper left panel of Fig. 5, but for the local specific star formation rate, $\operatorname{SSFR}(R)=\Sigma_{\mathrm{SFR}}(R) / \mu_{\star}(R)$. The error bar shows the $1 \sigma$ dispersion in log SSFR for Sb galaxies. The gray dashed line at $s S F R=0.1 \mathrm{Gyr}^{-1}$ indicates the value adopted by Peng et al. (2010) as a threshold for separating star-forming galaxies from quiescent systems.

by the bulge and that the disk dominates outside 1 HLR, we can compare the sSFR values in these two morphological components through a ratio like $s S F R(R=0.1) / s S F R(R=1.5)$. For early type spirals ( $\mathrm{S} 0, \mathrm{Sa}, \mathrm{Sb})$, this exercise results in the sSFR of bulges being on average 0.40 dex smaller than in the disks. The difference is greater, $0.60 \mathrm{dex}$, for Sbc, while for later later types (Sc, Sd, with their small on non-existent bulges) it decreases to 0.24 dex. The sample dispersions around these values is $\sim 0.3$ dex.

As in the case of the global sSFR, the local one can also be expressed as a characteristic time scale of star formation, $\tau(R)=$ $\operatorname{sSFR}(R)^{-1}$ that, independently of IMF and cosmology, tells the period of time that the system needs to build its current stellarmass-forming stars at the present rate ${ }^{5}$. Measured at $R=1 \mathrm{HLR}$, $\tau$ ranges from $12.6 \mathrm{Gyr}$ in Sbc to $5 \mathrm{Gyr}$ in Sd galaxies. Early-type spirals ( $\mathrm{Sa}, \mathrm{Sb}$ ), S0, and E would all need more than the Hubble time to build their mass at their current SFR.

Figure 7 at $s S F R=0.1 \mathrm{Gyr}^{-1}$ shows the value adopted by Peng et al. (2010) as a threshold to separate star-forming galaxies from quiescent systems and the sSFR that galaxies should have to build their mass at the present rate during a Hubble time (approximated to $10 \mathrm{Gyr}$ ). The comparison of $0.1 \mathrm{Gyr}^{-1}$ with the sSFR profiles indicates that $\mathrm{Sd}, \mathrm{Sc}$, and the disks of $\mathrm{Sbc}$ are very actively forming new stars, while $\mathrm{Sa}$ and $\mathrm{Sb}$ galaxies and the bulges of Sbc, although still forming stars, are evolving to quiescent systems.

Finally, E and S0 have $\operatorname{SSFR}(R)$ values that are 10-100 times less than $0.1 \mathrm{Gyr}^{-1}$, with a steep increase outward. This suggests that quenching in these galaxies has progressed inside-out.

\footnotetext{
5 This standard reading of $\tau=s S F R^{-1}$ actually neglects the difference between the mass turned into stars and that which stays in stars (or remnants). Because the denominator in SSFR is the current stellar mass, a rigorous definition would require a $(1-\mathcal{R})^{-1}$ correction for the returned mass fraction $\mathcal{R}$, not important for the discussion at this point.
} 


\section{Discussion}

The central goal of this paper, the one embodied in its very title, was fulfilled in the previous section with the results on the radial profiles of $\Sigma_{\mathrm{SFR}}$ and sSFR for galaxies along the Hubble sequence (Figs. 5 to 7). In this final part we go beyond this point and examine a few related issues. First we take advantage of the volume corrections computed by Walcher et al. (2014) to extrapolate the SFR computed from our sample to a local Universe SFR density and how it breaks up into contributions from different Hubble types and radial regions (Sect. 6.1). Second, we re-express our results (both the radial profiles and the local Universe average) for SFR in terms of the birthrate parameter $b$ (Sect. 6.2). We then turn our eyes to the spatially resolved version of the global MSSF. Like the SFR and $M_{\star}$ for entire galaxies (Fig. 4), their surface densities $\Sigma_{\mathrm{SFR}}$ and $\mu_{\star}$ correlate strongly, with a morphology-related scatter (Sect. 6.3). Finally, we gather our results to formulate an empirical scenario that identifies the origin of the global MSSF (Sect. 6.4).

\subsection{The SFR volume density in the local Universe}

CALIFA, as for many other samples, is not limited in volume, but can be "volume-corrected" using the $V_{\max }$ method (Schmidt 1968). Here, $V_{\max }$ is the volume available per galaxy, calculated for a diameter-limited sample by assuming that the ratio between apparent and linear isophotal size of a galaxy only depends on its angular diameter distance (see Walcher et al. 2014 for details).

We use this method to extend our results to those expected for local Universe galaxies as a whole. In particular, we transform our SFR estimates into the volume density of SFR, $\rho_{\mathrm{SFR}}$, by adding $S F R / V_{\max }$ for our galaxies and correcting the result by $\times 937 / 414$, the ratio of galaxies in the mother sample to those used in this paper ${ }^{6}$. CALIFA is a local sample, so there is no need to correct for evolution over the lookback time spanned by its redshift limits.

This process yields $\rho_{\mathrm{SFR}}=0.0105 \pm 0.0008$ (random) $M_{\odot} \mathrm{yr}^{-1} \mathrm{Mpc}^{-3}$. Figure 8 places our estimate (black star) in the $\rho_{\mathrm{SFR}}$ vs. $z$ diagram, along with other values from the literature, coming from different samples and methods. The dashed lines show the evolution of $\rho_{\mathrm{SFR}}$ from Madau $\&$ Dickinson (2014), Hopkins \& Beacom (2006), and Fardal et al. (2007). We also include the local $\rho_{\mathrm{SFR}}$ from the compilation of Gunawardhana et al. (2015, 2013), and the results obtained by Panter et al. (2003) from the fossil record method applied to the SDSS data. When necessary, the literature results are scaled to a Salpeter IMF. Our estimate is smaller by 0.15 dex and higher by 0.05 dex than the values at $z=0$ from Madau \& Dickinson (2014) and Fardal et al. (2007), respectively. It is also in excellent agreement with the $z<0.1$ estimates compiled by Gunawardhana et al. (2015, 2013), which average $0.0109 M_{\odot} \mathrm{yr}^{-1} \mathrm{Mpc}^{-3}$.

Obviously, the above refers to integrated measurements, which in our case is tantamount to collapsing all our 11894 radial points into a single number. To explore our data better, Fig. 8 also shows the contribution to the overall $\rho_{\mathrm{SFR}}$ from the different morphological types, plotted as stars (color-coded by their morphology). It is clear that $\mathrm{Sbc}, \mathrm{Sc}$, and $\mathrm{Sd}$ galaxies dominate the $\rho_{\text {SFR }}$ budget. Together they contribute $\sim 75 \%$ of $\rho_{\text {SFR }}$, despite accounting for only $\sim 24 \%$ of the stellar mass volume density of

\footnotetext{
6 For this analysis we exclude NGC 4676B and NGC 5947 because they do not belong to the original CALIFA sample and have no associated $V_{\max }$ estimates.
}

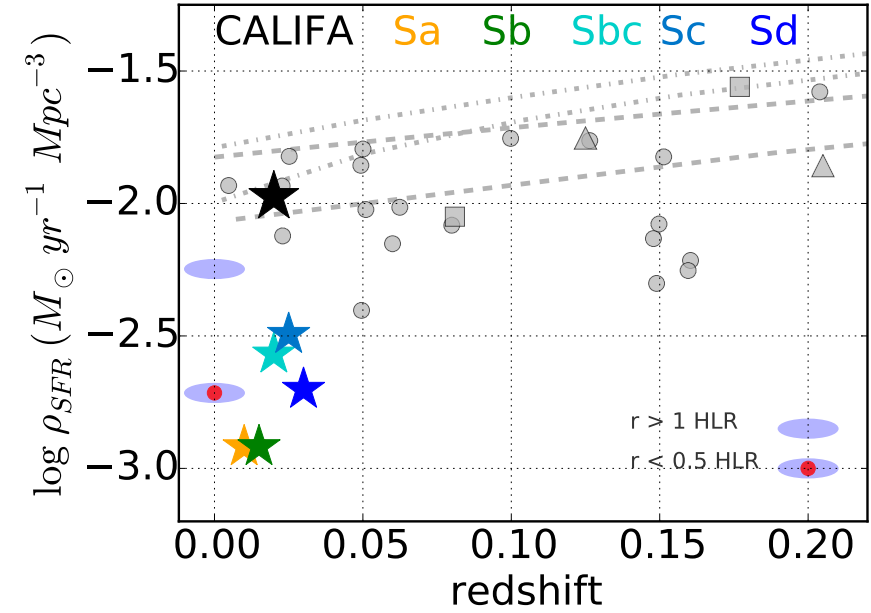

Fig. 8. Star formation rate density in the present study (black star). Colored stars represent the contribution of each morphological type to the total star formation rate density $\left(\rho_{\mathrm{SFR}}\right)$. The blue ellipse represents the contribution to $\rho_{\mathrm{SFR}}$ of the regions outside 1 HLR; and the red-dot blue ellipse represents the contribution to $\rho_{\text {SFR }}$ of the regions inside 0.5 HLR. Other results are from recent determinations by Gunawardhana et al. (2013; 2015; gray triangles) and their compilation (gray points), and the redshift evolution of $\rho_{\mathrm{SFR}}$ from Hopkins \& Beacom (2006; the top two gray dotted lines are $\pm 1 \sigma$ of their relation); Madau \& Dickinson (2014; middle gray dashed line); Fardal et al. (2007; bottom gray dashed line); and from the fossil record method applied to SDSS data by Panter et al. (2003; gray squares). When needed, literature values have been scaled to a Salpeter IMF.

the local Universe $\left(\rho_{\star}\right.$, computed following the same methodology). In contrast, $\mathrm{Sa}$ and $\mathrm{Sb}$ galaxies contribute $\sim 22 \%$ to $\rho_{\mathrm{SFR}}$ and $\sim 33 \%$ to $\rho_{\star}$, while E and S0 add less than $2 \%$ to $\rho_{\text {SFR }}$ but $43 \%$ to $\rho_{\star}$.

In terms of spatial origin, $53 \%$ of $\rho_{\mathrm{SFR}}$ comes from the regions outwards of $1 \mathrm{HLR}, 29 \%$ from $0.5<R<1 \mathrm{HLR}$, and $18 \%$ from the inner $0.5 \mathrm{HLR}$. In contrast, the $\rho_{\star}$ budget for these same regions are 40, 25 and 35\%, respectively. Most of the ongoing star formation thus occurs outside the centers, in disk - dominated regions, while the stellar mass is more evenly distributed with radius. If we take the half mass radius (HMR) as reference, which is typically $0.8 \times$ the HLR (González Delgado et al. 2015), we find that only $35 \%$ of $\rho_{\mathrm{SFR}}$ comes from the regions inside the central $1 \mathrm{HMR}$, suggesting again that most of the star formation density comes from the disk-dominated regions.

\subsection{The birthrate parameter}

It is often useful to consider the SFR in relation to some fiducial value, instead of in absolute units. A classical example is the birthrate parameter, $b$, that measures the current SFR of a system with respect to its lifetime average, $\langle\mathrm{SFR}\rangle_{\text {cosmic }}$ (Kennicutt 1983b; Scalo \& Struck-Marcell 1986). This parameter conveniently separates galaxies with declining SFRs $(b<1)$ from those with SFR increasing $(b>1)$ from past to present. Here, $b$ and SSFR are related by

$b=S F R /\langle S F R\rangle_{\text {cosmic }}=s S F R t_{\infty}(1-\mathcal{R})$

where $t_{\infty}$ is the time over which the galaxy has formed stars ${ }^{7}$, and $\mathcal{R}$ denotes the fraction of the mass initially turned into stars that is returned to the interstellar medium by stellar evolution. In

\footnotetext{
$7 t_{\infty}=t_{\mathrm{H}}(z)-t_{\text {form }}$, where $t_{\mathrm{H}}(z)$ is the Hubble time at redshift $z$ and $t_{\text {form }}$
} is the time of formation. 


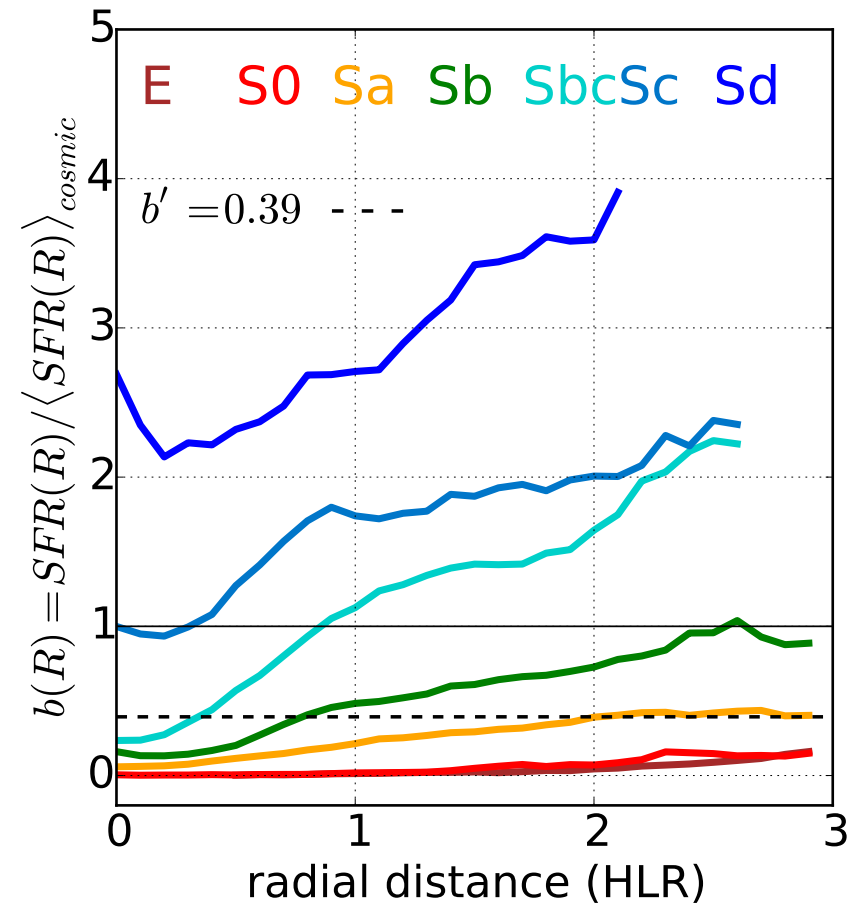

Fig. 9. Radial profiles of the birthrate parameter, $b(R)$, averaged in seven Hubble type bins; $b(R)$ compares the present to the past-average SFR at radius $R$ (cf. Eq. (4)). The dashed and solid black lines indicate our (volume-corrected) value for the local Universe and the reference value $b(R)=1$, respectively.

practice, $b=10.08 s \operatorname{SFR}\left(\mathrm{Gyr}^{-1}\right)$ for $t_{\infty}=14 \mathrm{Gyr}$ and $(1-\mathcal{R})=$ 0.72 (average over our sample and the Salpeter IMF assumed in the models).

A volume-corrected value of $b$ representative of the local Universe can be obtained from

$b^{\prime}=\frac{\sum_{i} S F R_{i} V_{\max , i}^{-1}}{\sum_{i}\langle S F R\rangle_{\operatorname{cosmic}, i} V_{\max , i}^{-1}}$.

We find that $b^{\prime}=0.39 \pm 0.03$ (random), meaning that the presentday Universe is forming stars at a little over one-third of its past average rate.

As for $\rho_{\text {SFR }}$ (Sect. 6.1), though useful, this one-numbersummary of the star formation history of the Universe as a whole averages over the richness of information in CALIFA data. Our spatially resolved observations allow for definitions of $b$ that take its variation within galaxies into account (Cid Fernandes et al. 2013), the simplest of which is

$b(R)=\Sigma_{\mathrm{SFR}}(R) /\left\langle\Sigma_{\mathrm{SFR}}(R)\right\rangle_{\mathrm{cosmic}}$.

This radial profile $b(R)$ behaves exactly like the global $b$ of Eq. (2). There is, however, a relevant assumption implicit in this comparison of past and present as a function to radius, namely, that (statistically) stars do not move too far from their birthplaces during their lives.

Figure 9 shows our results for $b(R)$ for our seven Hubble types. Clear and systematic trends are identified with both radial distance and morphology. First, $b(R)$ increases outward, as expected from the inside-out growth of galaxies (Pérez et al. 2013; González Delgado et al. 2014b, 2015; Sánchez-Blázquez et al. 2014; Sánchez et al. 2014). Second, $b(R)$ scales in amplitude with Hubble type, increasing from early to late spirals. The $\mathrm{Sd}$ and Sc galaxies are currently forming stars faster than in the past at all radii. The disks $(R>1$ HLR) of Sbc galaxies also show $b(R)>1$, but their bulges are forming stars at lower rates than in the past. Sb and earlier types have $b(R)<1$ throughout their disks and bulges.

Finally, we note that spheroids (E, S0, and the inner regions of early type spirals, presumably associated with bulges) all have $b<b^{\prime}$. Star formation has thus stopped (or been quenched) some ime ago. Most regions in Sa have $b(R)<0.39$, so, even though these galaxies are still forming stars, they are located in the transition between the MSSF and the quenched cloud.

\subsection{The local main sequence of star formation}

CALIFA is ideally suited to investigating the roles of global and local properties controlling the MSSF. In fact, using the spatially resolved $\mathrm{H} \alpha$ flux of more than 500 CALIFA galaxies, Cano-Díaz et al. (2016) have recently found that the $\mathrm{H} \alpha$-based $\Sigma_{\mathrm{SFR}}$ correlates with the stellar mass surface density, $\mu_{\star}$, and that the slope and dispersion of this local MSSF are similar to those of the global $S F R-M_{\star}$ relation. A local MSSF relation has also been also reported by Wuyts et al. (2013) in a sample of massive star-forming galaxies at $z \sim 1$ through spatially resolved $\mathrm{H} \alpha$ images provided by HST. Section 4 presented our version of the global MSSF (see Fig. 4). Here we use our radial profile data to investigate the local relation.

Figure 10 plots $\Sigma_{\mathrm{SFR}}(R)$ against $\mu_{\star}(R)$ for the nearly 12 thousand radial bins in our 416 galaxies. The plot is ultimately a collection of $416 \Sigma_{\mathrm{SFR}}(R)$ profiles where the radial coordinate is replaced by $\mu_{\star}(R)$. Clearly, our STARLIGHT-based $\Sigma_{\mathrm{SFR}}(R)$ and $\mu_{\star}(R)$ values correlate. Dotted diagonals indicate lines of constant sSFR. An eyeball comparison of these lines with the data already hints that, like the global one, the local MSSF is sublinear.

Large white circles in the lefthand panel of Fig. 10 show the mean $\Sigma_{\mathrm{SFR}}$ in 0.2 dex wide bins in $\mu_{\star}$. The scatter around this mean relation is visibly related to morphology, as further illustrated by the mean relations obtained for $\mathrm{Sa}$ and $\mathrm{Sc}$ galaxies. The increase in $\Sigma_{\mathrm{SFR}}$ at fixed $\mu_{\star}$ from early to late types is another manifestation of our earlier finding that the $\operatorname{sSFR}(R)$ profiles scale with Hubble type (Fig. 7).

Galaxies of different morphologies thus seem to follow roughly parallel local MSSF relations of the type $\log \Sigma_{\mathrm{SFR}}=$ $\alpha \log \mu_{\star}+\beta$, with similar logarithmic slopes $(\alpha)$ but zero points $(\beta)$ increasing steadily from early to late types. This behavior prompted us to follow a two-step approach to estimate $\alpha$ and $\beta$. First, scale effects are removed by rescaling both $\Sigma_{\mathrm{SFR}}$ and $\mu_{\star}$ for each galaxy by their corresponding values at $R=1$ HLR. The value of $\alpha$ obtained in this way is then used to derive $\beta$ as the average of $\log \Sigma_{\mathrm{SFR}}-\alpha \log \mu_{\star}^{8}$.

For the whole data set we obtain $\alpha=0.70 \pm 0.01$ and $\beta=-0.53 \pm 0.02$, with an rms dispersion of 0.27 dex. We have also carried out fits weighting each point by the $V_{\max }^{-1}$ value of its host galaxy ${ }^{9}$, which gives the slope and zero point the status of being representative of the local Universe. The parameters for this alternative fit are $\alpha^{\prime}=0.84 \pm 0.01$ and $\beta^{\prime}=-0.85 \pm 0.03$. This fit is shown as a dashed black and gray line in the righthand panel of Fig. 10, which repeats our local MSSF, but now coloring each radial bin of each galaxy by its contribution to the total SFR cosmic density in the local Universe $\left(\rho_{\text {SFR }}\right)$.

8 Units of $M_{\odot} \mathrm{yr}^{-1} \mathrm{pc}^{-2}$ and $M_{\odot} \mathrm{pc}^{-2}$ are assumed throughout.
9 In these "volume-corrected fits" the weight attributed to all radial
bins of the $i$ th galaxy is $w_{i}=V_{\max , i}^{-1} / \sum_{j} V_{\max , j}^{-1}$, where the sum runs over bins of the $i$ th
all galaxies. 


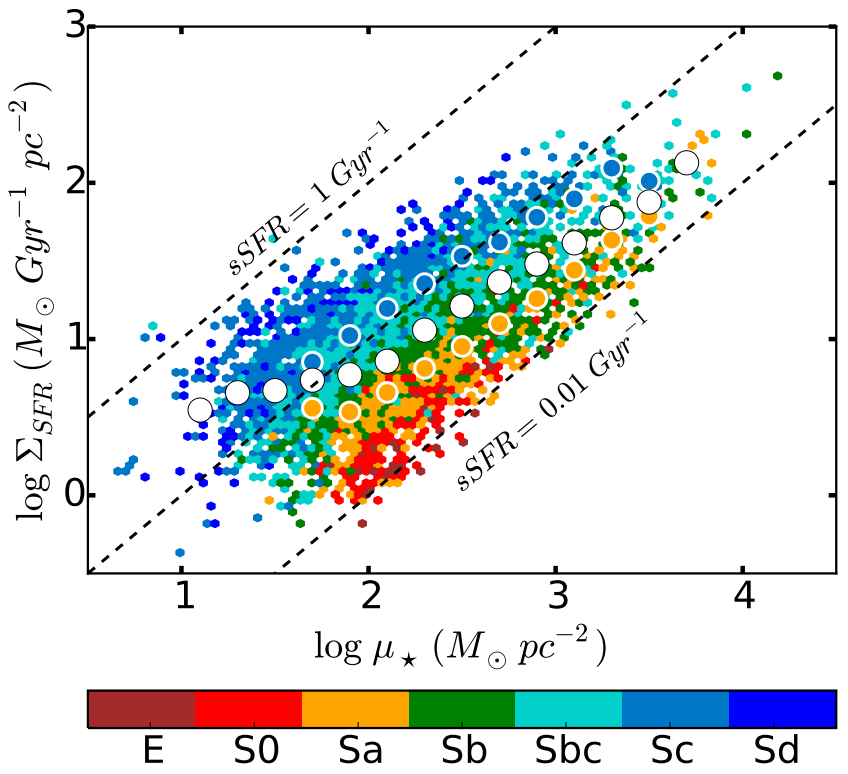

Hubble type
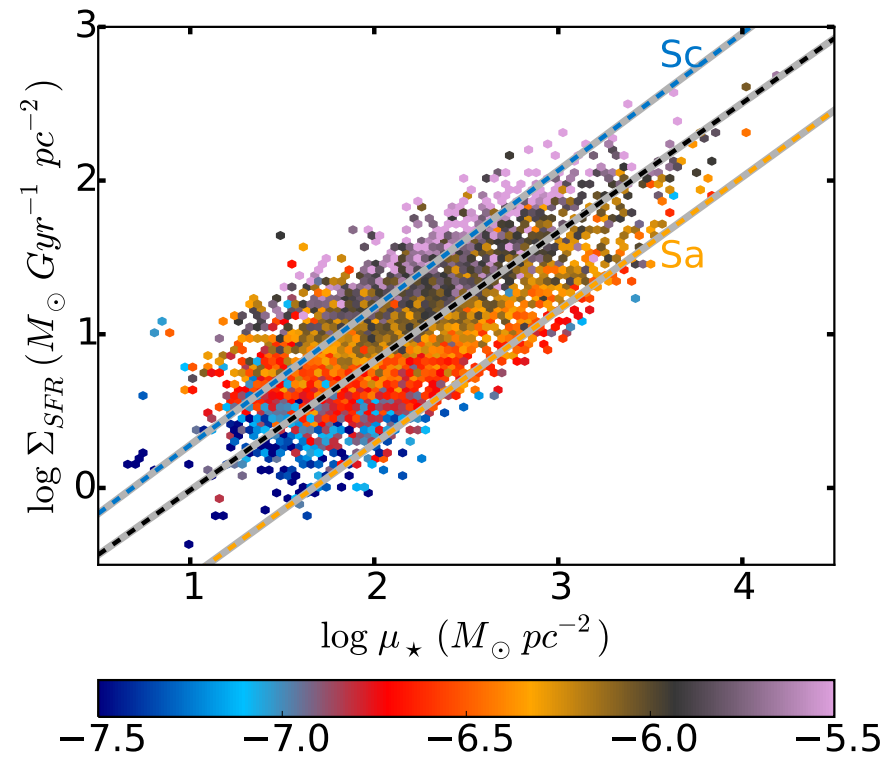

$\log \rho_{S F R}\left(M_{\odot} y r^{-1} M p c^{-3}\right)$

Fig. 10. Left: local star-forming main sequence $\mu_{\star}-\Sigma_{\mathrm{SFR}}$ relation, color-coded by morphology. Large white circles show the mean (i.e., $\mu_{\star}$-binned) relation for all points; orange and blue circles show $\mu_{\star}-\Sigma_{\mathrm{SFR}}$ for Sa and Sc galaxies, respectively. Only points for which $x_{Y}>3.4 \%$ are included. Diagonal dashed black lines are loci of constant $s S F R=0.01,0.1$, and $1 \mathrm{Gyr}^{-1}$. Right: the same $\mu_{\star}-\Sigma_{\mathrm{SFR}}$ distribution as in the left panel but with points weighted with $\log \rho_{\mathrm{SFR}}$ (E and S0 are excluded). Lines show the linear fits (for $\alpha^{\prime}$ and $\beta^{\prime}$ ) to the points in Sa and Sc galaxies, and for all spirals (black-gray).

Table 3. Parameters of $\log \Sigma_{\mathrm{SFR}}\left(M_{\odot} \mathrm{yr}^{-1} \mathrm{pc}^{-2}\right)=\alpha \log \mu_{\star}\left(M_{\odot} \mathrm{pc}^{-2}\right)+\beta$ fits to the local MSSF to spirals of different types.

\begin{tabular}{lcccccc}
\hline \hline Morph. & All & Sa & Sb & Sbc & Sc & Sd \\
\hline$\alpha$ & 0.70 & 0.60 & 0.68 & 0.70 & 0.79 & 0.85 \\
$\alpha^{\prime}$ & 0.84 & 0.87 & 0.79 & 0.78 & 0.89 & 0.80 \\
$\beta$ & -0.55 & -0.70 & -0.64 & -0.32 & -0.41 & -0.39 \\
$\beta^{\prime}$ & -0.85 & -1.45 & -0.93 & -0.50 & -0.60 & -0.30 \\
\hline
\end{tabular}

These fits describe the local MSSF well as a whole, but completely overlook the evident role of morphology. It is thus more appropriate to fit the relation for different Hubble types, in analogy with what was done in Table 2 for the global MSSF. Table 3 lists the $\alpha$ and $\beta$ values obtained subdividing the sample in morphology. Coefficients for the $V_{\max }$-weighted fits $\left(\alpha^{\prime}\right.$ and $\left.\beta^{\prime}\right)$ are also given. Dashed blue/gray and orange/gray lines in the righthand panel of Fig. 10 show the fitted relations for Sc and Sa galaxies, respectively.

Inspection of the results in Table 3 shows that, as anticipated by a visual assessment of the local MSSF, slopes are indeed fairly similar for all types, while $\beta$ increases monotonically from early to late types. In all cases we obtain $\alpha<1$. While the mixture of morphological types certainly explains part of the sublinearity of both the global and local MSSF when lumping all sources together, this result indicates that the local MSSF is sublinear even for fixed Hubble type. Our values of $\alpha\left(\alpha^{\prime}\right)=0.68(0.79)$ for $\mathrm{Sb}$ and $0.79(0.89)$ for Sc galaxies bracket the slope of 0.72 derived by Cano-Díaz et al. (2016) for the $\mathrm{H} \alpha$-based local MSSF relation.

Our own previous work has shown that $\mu_{\star}$ is an effective tracer of local stellar population properties. Both mean stellar ages (González Delgado et al. 2014b) and metallicities (González Delgado et al. 2014a) correlate well with $\mu_{\star}$, and this work shows that $\Sigma_{\text {SFR }}$ also follows this pattern. These previous studies reveal that the overall balance between local ( $\mu_{\star}$-driven) and global $\left(M_{\star}\right.$-driven) effects varies with the location within the galaxy. While in disks $\mu_{\star}$ regulates the mean stellar ages and metallicities, it plays a minor role in spheroids (bulges and elliptical galaxies), whose chemical enrichment happened much faster and earlier than in disks. How does the local MSSF relation found in this work fit into this general scheme?

On the one hand, we have seen that the local MSSF relation is mostly a disk phenomenon. In fact, it points to a density dependence of the SFR law akin to what was proposed by Schmidt (1959) and Kennicutt (1998), where the gas density sets the rate at which stars form. On the other hand, the clear role of Hubble type in defining the offset around the overall $\Sigma_{\mathrm{SFR}}-\mu_{\star}$ relation suggests that some global morphology-related property modulates the local sSFR. Gas content is an obvious candidate hidden variable in this context (Roberts \& Haynes 1994; Tacconi et al. 2013).

Alternatively (or complementarily), the modulation of the $\Sigma_{\mathrm{SFR}}-\mu_{\star}$ relation with Hubble type may reflect the effect of a "morphological quenching". In González Delgado et al. (2015), we have found that, for the same $M_{\star}$, early type galaxies are older than later types, both globally and in the disk, and that this ranking is maintained with radial distance. This gradual age change from spheroidals to $\mathrm{Sa}$ and to late spirals reflects the change in sSFR with Hubble type and can be interpreted as a consequence of the mechanism building the bulge. The steep potential well induced by the formation of a large spheroid component stabilizes the disk, cutting the supply of the gas and preventing its local fragmentation into bound, star-forming clumps (Martig et al. 2009). This effect should thus be more significant in E and S0 and gradually decrease from Sa to Sb. Later types, Sc and Sd, where the bulge (if present) may be formed by secular processes, may not be affected by this morphological quenching. 


\subsection{The relation between local and global MSSFs}

The results reported throughout this paper give plenty of material to explore in relation to galaxy structure and evolution studies. In this final section we develop some simple math relating the local and global MSSF relations.

The global (i.e., spatially integrated) SFR and stellar mass of a galaxy relate to the local properties through

$S F R=2 \pi \int \Sigma_{\mathrm{SFR}}(R) R \mathrm{~d} R=2 \pi R_{0}^{2} \Sigma_{\mathrm{SFR}}\left(R_{0}\right) s_{\Sigma}$
$M_{\star}=2 \pi \int \mu_{\star}(R) R \mathrm{~d} R=2 \pi R_{0}^{2} \mu_{\star}\left(R_{0}\right) s_{\mu}$,

where we have denoted HLR by $R_{0}$ for convenience, and

$s_{\Sigma} \equiv \int \frac{\Sigma_{\mathrm{SFR}}(R)}{\Sigma_{\mathrm{SFR}}\left(R_{0}\right)} \frac{R}{R_{0}} \frac{\mathrm{d} R}{R_{0}}$

$s_{\mu} \equiv \int \frac{\mu_{\star}(R)}{\mu_{\star}\left(R_{0}\right)} \frac{R}{R_{0}} \frac{\mathrm{d} R}{R_{0}}$

are shape factors of order unity. Equations (5) and (6) lead to

$S F R=\frac{s_{\Sigma}}{s_{\mu}} \frac{\Sigma_{\mathrm{SFR}}\left(R_{0}\right)}{\mu_{\star}\left(R_{0}\right)} M_{\star}$,

which predicts the global MSSF relation in terms of spatially resolved properties ${ }^{10}$.

Direct integration of the profiles yields a $s_{\Sigma} / s_{\mu}$ ratio of typically $0.9 \pm 0.4$ for our spirals (average and dispersion) and a very weak $\left(\propto M_{\star}^{-0.07}\right)$ trend with mass. Relevant deviations from a linear global MSSF must therefore come from variations in $\operatorname{SSFR}\left(R_{0}\right)=\Sigma_{\mathrm{SFR}}\left(R_{0}\right) / \mu_{\star}\left(R_{0}\right)$ with $M_{\star}$.

Figure 7 shows that $\operatorname{SSFR}\left(R_{0}\right)$ increases systematically toward later type spirals, indicating an anti-correlation with stellar mass, hence a sublinear predicted global MSSF. More quantitatively, recalling that $\Sigma_{\mathrm{SFR}} \propto \mu_{\star}^{\alpha}$ from our local MSSF relation and that $\mu_{\star}\left(R_{0}\right) \propto M_{\star}^{\gamma}$ with $\gamma \sim 0.5$ (González Delgado et al. 2014b), the predicted relation goes as $S F R \propto M_{\star}^{1-\gamma(1-\alpha)}$. For $\alpha$ between 0.70 and 0.84 (Table 3 ), and correcting for the mild trend of $s_{\Sigma} / s_{\mu}$ with mass, the predicted logarithmic slope of the global MSSF is in the 0.78-0.85 range, in good agreement with Renzini \& Peng (2015) and Cano-Díaz et al. (2016).

We close by noting that it is plausible to conclude from this analysis that the sublinearity of the local MSSF $(\alpha<1)$ is what causes sublinearity of the global $\mathrm{MSSF}^{11}$. A caveat in this tempting local $\rightarrow$ global argument is that it uses $\mu_{\star}$ to trace the local SFR density, whereas gas, not stars, is the actual fuel of star formation. We thus postpone further analysis of this issue to future work involving gas density estimates.

\section{Summary and conclusions}

We analyzed the stellar population properties of 416 galaxies observed by CALIFA at the $3.5 \mathrm{~m}$ telescope in Calar Alto, to investigate the trends of the recent star formation rate with radial distance and as a function of Hubble type. The sample includes ellipticals, S0, and spirals all the way from Sa to Sd,

\footnotetext{
10 Equation (9) can be written more compactly as $s S F=\frac{s_{\Sigma}}{s_{\mu}} S S F R\left(R_{0}\right)$, where the lefthand side is the global (spatially integrated) SSFR.

11 We clarify that the sublinearity we refer to here is not the one resulting from mixing galaxies of different morphologies in a same sample, but the one found when fitting the global MSSF at fixed Hubble type (i.e., the $a<1$ slopes in Table 2).
}

covering a stellar mass range from $\sim 10^{9}$ to $7 \times 10^{11} M_{\odot}$ (for a Salpeter IMF). A full spectral fitting analysis was performed using the STARLIGHT code and a combination of SSP spectra from González Delgado et al. (2005) plus Vazdekis et al. (2010). Our PyCASso pipeline was used to process the spectral fitting results to produce maps of the recent star formation rate (SFR, averaged over the past $32 \mathrm{Myr}$ ), and the stellar mass surface density $\left(\mu_{\star}\right)$. For each galaxy, the maps are azimuthally averaged to produce radial profiles (in units of the half light radius, HLR) of the SFR surface density, $\Sigma_{\mathrm{SFR}}(R)$, and the corresponding local specific SFR, $\operatorname{SSFR}(R)=\Sigma_{\mathrm{SFR}}(R) / \mu_{\star}(R)$. Variations in the traditional birthrate parameter, $b$, are obtained to compare the present and the past SFRs at different radial positions. The radial profiles are stacked as a function of Hubble type and of galaxy mass to identify the main trends.

Our main results are:

1. Spiral galaxies have declining $\Sigma_{\mathrm{SFR}}(R)$ profiles, with a relatively narrow range of $\Sigma_{\mathrm{SFR}}$ values at any given radial distance. At $R=1 \mathrm{HLR}$, the $\Sigma_{\mathrm{SFR}}$ is typically $20 M_{\odot} \mathrm{Gyr}^{-1} \mathrm{pc}^{-2}$, with a factor of two dispersion. Spirals with $M_{\star} \lesssim 4 \times$ $10^{10} M_{\odot}$ have $\Sigma_{\mathrm{SFR}}(R)$ profiles that are very similar and independent of Hubble type and galaxy mass. Above $4 \times 10^{10} M_{\odot}$, the $\Sigma_{\mathrm{SFR}}(R)$ profiles are slightly more dispersed. This is a remarkable result considering that the sample covers two orders of magnitude in $M_{\star}$ and all Hubble types. Ultimately, it is the constancy of $\Sigma_{\mathrm{SFR}}$ that, coupled to the $\mu_{\star}-M_{\star}$ relation, makes the MSSF a tight sequence.

2. In contrast, $\mathrm{E}$ and $\mathrm{S} 0$ galaxies have $\Sigma_{\mathrm{SFR}}(R)$ that are at all radii significantly depressed with respect to spirals, with flat $\Sigma_{\mathrm{SFR}} \sim 1 M_{\odot} \mathrm{Gyr}^{-1} \mathrm{pc}^{-2}$ profiles, and with a large uncertainty.

3. Expressed in units of the lifetime-averaged SFR intensity at each location, the present $\Sigma_{\mathrm{SFR}}(R)$ is currently lower in $\mathrm{E}, \mathrm{S} 0$, and early type spirals ( $\mathrm{Sa}$ and $\mathrm{Sb}$ ), but higher in later spirals (Sc and $\mathrm{Sd}$ ). Sbc galaxies seem to be the transition type in which "bulges" (central $\sim 1$ HLR) have already suppressed or quenched their star formation activity, as in Sa and Sb, but their disks are still forming new stars at a rate similar to the past.

4. The local $s S F R=\Sigma_{\mathrm{SFR}} / \mu_{\star}$ shows radial profiles that increase outwards and scale with Hubble type from $\mathrm{Sa}$ to $\mathrm{Sd}$. This behavior is preserved at any given $R$. This quantity, which relates the present and the past star formation rate locally, is orders of magnitude smaller in E and S0 than in spirals. The characteristic time scale of star formation given by $s S R^{-1}$ in spirals ranges from 12.6 Gyr in Sbc to 5 Gry in Sd galaxies. Early type spirals (Sa, Sb) and spheroidals (E, S0) would need more than a Hubble time to build their current stellar mass at their recent SFR.

5. The slope of $\operatorname{sSFR}(R)$ in the inner 1 HLR is steeper than outwards. This behavior with radial distance suggests that galaxies are quenched inside-out and that this process is faster in the central part (dominated by the bulge) than in the disk.

6. The CALIFA sample is well suited to computing the SFR density in the local Universe with a value $\rho_{\mathrm{SFR}}=0.0105 \pm$ 0.0008 (random) $M_{\odot} \mathrm{yr}^{-1} \mathrm{Mpc}^{-3}$ (for a Salpeter IMF), in excellent agreement with previous estimates from completely difference methods and data. We find that the majority of the star formation at $z=0$ take place in Sbc, Sc, and Sd galaxies with masses below $10^{11} M_{\odot}$. In terms of spatial distribution, most of the star formation is occurring outside galaxy centers, in regions that are mainly in the disks of spirals. 
7. The volume average birthrate parameter, $b^{\prime}=0.39$, suggests that the present-day Universe is forming stars at about onethird of its past average rate. E, S0, and the bulge of early type spirals have $b<0.39$, thus contributing little to the present SFR of the Universe. The disks (regions outside 1 HLR) of Sbc, and Sc, and Sd galaxies, all with $b>1$, dominate the present star formation of the Universe.

8. Galaxy mass and morphology, in particular the formation of a spheroidal component, play a relevant role in depressing/quenching the star formation in galaxies. Galaxies dominated by the spheroidal component, E and S0 in our sample, are all quiescent. Disk-dominated galaxies (Sbc, $\mathrm{Sc}, \mathrm{Sd})$ are very actively forming stars with a rate per unit mass that decreases with $M_{\star}$.

9. There is tight relation between the local values of $\mu_{\star}$ and $\Sigma_{\text {SFR }}$, defining a local main sequence of star-forming regions with slope $\sim 0.8$ and a scatter strongly related to Hubble type. This relation is tighter than the global main sequence relation between SFR and $M_{\star}$ once morphology-related offsets are accounted for. This suggests that local processes are important in determining the star formation in a galaxy, possibly due to a density dependence of the SFR law. The shut down of the star formation is more related to global processes, such as the formation of a spheroidal component. These findings agree with our previous analysis, which showed that the mean stellar ages and metallicity are mainly governed by local processes $\left(\mu_{\star}\right.$-driven) in disks and by global processes in spheroids.

Thanks to the uniqueness of CALIFA data and the homogeneity of our analysis, we were able to, for the first time, characterize the radial structure of the SFR along the Hubble sequence. This octogenarian sequence, by the way, has once again demonstrated its usefulness as a way to organize galaxies in terms of their spatially resolved properties. Our previous work showed the systematic behavior of $\mu_{\star}$, mean stellar ages, and metallicity with Hubble type, while this paper showed that the same also applies to the SFR and related quantities.

The large file FoV of PPaK allows us to cover galaxies in their entire optical extent, a design feature of CALIFA that eliminates aperture-related biases in the derivation of galaxy properties. Furthermore, the well-defined selection function of the survey allows for reliable volume corrections. In fact, an important "byproduct" of this study is that it exemplifies how well these corrections work, as demonstrated by the excellent agreement between our estimate for the $\rho_{\text {SFR }}$ of the local Universe and independent determinations from large galaxy surveys. This validates and reinforces the statistical approach to CALIFA followed in this, previous, and future papers in this series.

Acknowledgements. CALIFA is the first legacy survey carried out at Calar Alto. The CALIFA collaboration would like to thank the IAA-CSIC and MPIA-MPG as major partners of the observatory, and CAHA itself, for the unique access to telescope time and support in manpower and infrastructures. We also thank the CAHA staff for the dedication to this project. Support from the Spanish Ministerio de Economía y Competitividad, through projects AYA2014-57490P, AYA2010-15081, and Junta de Andalucía FQ1580, AYA2010-22111-C03-03, AYA2010-10904E, AYA2013-42227P, RyC-2011-09461, AYA2013-47742-C43-P, EU SELGIFS exchange program FP7-PEOPLE-2013-IRSES-612701, and CONACYT-125180 and DGAPA-IA100815. We also thank the Viabilidad, Diseño, Acceso y Mejora funding program, ICTS-2009-10, for funding the data acquisition of this project. A.L.d.A., E.A.D.L. and R.C.F. acknowledges the hospitality of the IAA and the support of CAPES and CNPq. R.G.D. acknowledges the support of $\mathrm{CNPq}$ (Brazil) through Programa Ciência sem Fronteiras (401452/2012-3). C.J.W. acknowledges support through the Marie Curie Caree Integration Grant 303912. We thank the IAA Computing group for their support, and the referee for useful comments.

\section{References}

Abazajian, K., Adelman-McCarthy, J. K., Agüeros, M. A., et al. 2003, AJ, 126, 2081

Asari, N. V., Cid Fernandes, R., Stasińska, G., et al. 2007, MNRAS, 381, 263

Ascasibar, Y., \& Sánchez Almeida, J. 2011, MNRAS, 415, 2417

Baldry, I. K., Glazebrook, K., Brinkmann, J., et al. 2004, ApJ, 600, 681

Bica, E. 1988, A\&A, 195, 76

Bica, E., Alloin, D., \& Schmitt, H. R. 1994, A\&A, 283, 805

Binette, L., Magris, C. G., Stasińska, G., \& Bruzual, A. G. 1994, A\&A, 292, 13

Blanton, M. R., \& Moustakas, J. 2009, ARA\&A, 47, 159

Blanton, M. R., Hogg, D. W., Bahcall, N. A., et al. 2003, ApJ, 592, 819

Brinchmann, J., Charlot, S., White, S. D. M., et al. 2004, MNRAS, 351, 1151

Bruzual, G., \& Charlot, S. 2003, MNRAS, 344, 1000

Bryant, J. J., Owers, M. S., Robotham, A. S. G., et al. 2015, MNRAS, 447, 2857

Bundy, K., Bershady, M. A., Law, D. R., et al. 2015, ApJ, 798, 7

Cano-Díaz, M., Sánchez, S. F., Zibetti, S., et al. 2016, ApJ, 821, L26

Cappellari, M., \& Copin, Y. 2003, MNRAS, 342, 345

Cappellari, M., Emsellem, E., Krajnović, D., et al. 2011, MNRAS, 413, 813

Casado, J., Ascasibar, Y., Gavilán, M., et al. 2015, MNRAS, 451, 888

Catalán-Torrecilla, C., Gil de Paz, A., Castillo-Morales, A., et al. 2015, A\&A, 584, A87

Chabrier, G. 2003, PASP, 115, 763

Chang, Y.-Y., van der Wel, A., da Cunha, E., \& Rix, H.-W. 2015, ApJS, 219, 8

Charbonnel, C., Meynet, G., Maeder, A., Schaller, G., \& Schaerer, D. 1993, A\&AS, 101, 415

Cid Fernandes, R., \& González Delgado, R. M. 2010, MNRAS, 403, 780

Cid Fernandes, R., Heckman, T., Schmitt, H., González Delgado, R. M., \& Storchi-Bergmann, T. 2001, ApJ, 558, 81

Cid Fernandes, R., González Delgado, R. M., Schmitt, H., et al. 2004, ApJ, 605, 105

Cid Fernandes, R., Mateus, A., Sodré, L., Stasińska, G., \& Gomes, J. M. 2005, MNRAS, 358, 363

Cid Fernandes, R., Stasińska, G., Mateus, A., \& Vale Asari, N. 2011, MNRAS, 413, 1687

Cid Fernandes, R., Pérez, E., García Benito, R., et al. 2013, A\&A, 557, A86

Cid Fernandes, R., González Delgado, R. M., García Benito, R., et al. 2014, A\&A, 561, A130

Conroy, C. 2013, ARA\&A, 51, 393

Daddi, E., Dickinson, M., Morrison, G., et al. 2007, ApJ, 670, 156

Elbaz, D., Daddi, E., Le Borgne, D., et al. 2007, A\&A, 468, 33

Elbaz, D., Dickinson, M., Hwang, H. S., et al. 2011, A\&A, 533, A119

Faber, S. M., Willmer, C. N. A., Wolf, C., et al. 2007, ApJ, 665, 265

Fardal, M. A., Katz, N., Weinberg, D. H., \& Davé, R. 2007, MNRAS, 379, 985

Flores-Fajardo, N., Morisset, C., Stasińska, G., \& Binette, L. 2011, MNRAS, 415,2182

Gallagher, III, J. S., Hunter, D. A., \& Tutukov, A. V. 1984, ApJ, 284, 544 García-Benito, R., Zibetti, S., Sánchez, S. F., et al. 2015, A\&A, 576, A135 Girardi, L., Bressan, A., Bertelli, G., \& Chiosi, C. 2000, A\&AS, 141, 371 Gomes, J. M., Papaderos, P., Kehrig, C., et al. 2015, A\&A, 588, A68 Gomes, J. M., Papaderos, P., Vílchez, J. M., et al. 2016a, A\&A, 586, A22 Gomes, J. M., Papaderos, P., Vílchez, J. M., et al. 2016b, A\&A, 585, A92 González Delgado, R. M., Cid Fernandes, R., Pérez, E., et al. 2004, ApJ, 605, 127

González Delgado, R. M., Cerviño, M., Martins, L. P., Leitherer, C., \& Hauschildt, P. H. 2005, MNRAS, 357, 945

González Delgado, R. M., Cid Fernandes, R., García-Benito, R., et al. 2014a, ApJ, 791, L16

González Delgado, R. M., Pérez, E., Cid Fernandes, R., et al. 2014b, A\&A, 562, A47

González Delgado, R. M., García-Benito, R., Pérez, E., et al. 2015, A\&A, 581, A103

Gunawardhana, M. L. P., Hopkins, A. M., Bland-Hawthorn, J., et al. 2013, MNRAS, 433, 2764

Gunawardhana, M. L. P., Hopkins, A. M., Taylor, E. N., et al. 2015, MNRAS, 447,875

Heavens, A., Panter, B., Jimenez, R., \& Dunlop, J. 2004, Nature, 428, 625

Heckman, T. M., Hoopes, C. G., Seibert, M., et al. 2005, ApJ, 619, L35

Holmberg, E. 1958, Meddelanden fran Lunds Astronomiska Observatorium Serie II, 136, 1

Hopkins, A. M., \& Beacom, J. F. 2006, ApJ, 651, 142

Hubble, E. 1936, ApJ, 84, 517

Husemann, B., Jahnke, K., Sánchez, S. F., et al. 2013, A\&A, 549, A87

Karim, A., Schinnerer, E., Martínez-Sansigre, A., et al. 2011, ApJ, 730, 61

Kauffmann, G., Heckman, T. M., White, S. D. M., et al. 2003, MNRAS, 341, 33

Kaviraj, S., Schawinski, K., Devriendt, J. E. G., et al. 2007, ApJS, 173, 619 
Kehrig, C., Monreal-Ibero, A., Papaderos, P., et al. 2012, A\&A, 540, A11 Kelz, A., Verheijen, M. A. W., Roth, M. M., et al. 2006, PASP, 118, 129 Kennicutt, R. C., \& Evans, N. J. 2012, ARA\&A, 50, 531

Kennicutt, Jr., R. C. 1983a, ApJ, 272, 54

Kennicutt, Jr., R. C. 1983b, ApJ, 272, 54

Kennicutt, Jr., R. C. 1998, ARA\&A, 36, 189

Koleva, M., Prugniel, P., de Rijcke, S., \& Zeilinger, W. W. 2011, MNRAS, 417, 1643

Leitherer, C., Schaerer, D., Goldader, J. D., et al. 1999, ApJS, 123, 3

Licquia, T. C., \& Newman, J. A. 2015, ApJ, 806, 96

Lilly, S. J., Carollo, C. M., Pipino, A., Renzini, A., \& Peng, Y. 2013, ApJ, 772, 119

Madau, P., \& Dickinson, M. 2014, ARA\&A, 52, 415

Maraston, C. 2005, MNRAS, 362, 799

Martig, M., Bournaud, F., Teyssier, R., \& Dekel, A. 2009, ApJ, 707, 250

Mateus, A., Sodré, L., Cid Fernandes, R., et al. 2006, MNRAS, 370, 721

McDermid, R. M., Alatalo, K., Blitz, L., et al. 2015, MNRAS, 448, 3484

Noeske, K. G., Faber, S. M., Weiner, B. J., et al. 2007, ApJ, 660, L47

Ocvirk, P. 2010, ApJ, 709, 88

Ocvirk, P., Pichon, C., Lançon, A., \& Thiébaut, E. 2006, MNRAS, 365, 74

Panter, B., Heavens, A. F., \& Jimenez, R. 2003, MNRAS, 343, 1145

Panter, B., Jimenez, R., Heavens, A. F., \& Charlot, S. 2008, MNRAS, 391, 111

Papaderos, P., Gomes, J. M., Vílchez, J. M., et al. 2013, A\&A, 555, L1

Peng, Y.-j., Lilly, S. J., Kovač, K., et al. 2010, ApJ, 721, 193

Pérez, E., Cid Fernandes, R., González Delgado, R. M., et al. 2013, ApJ, 764, L1

Renzini, A., \& Peng, Y.-j. 2015, ApJ, 801, L29

Roberts, M. S. 1963, ARA\&A, 1, 149

Roberts, M. S., \& Haynes, M. P. 1994, ARA\&A, 32, 115

Rodighiero, G., Daddi, E., Baronchelli, I., et al. 2011, ApJ, 739, L40

Roth, M. M., Kelz, A., Fechner, T., et al. 2005, PASP, 117, 620

Salim, S., Rich, R. M., Charlot, S., et al. 2007, ApJS, 173, 267

Sánchez, S. F., Kennicutt, R. C., Gil de Paz, A., et al. 2012, A\&A, 538, A8

Sánchez, S. F., Rosales-Ortega, F. F., Iglesias-Páramo, J., et al. 2014, A\&A, 563, A49
Sánchez, S. F., Pérez, E., Rosales-Ortega, F. F., et al. 2015, A\&A, 574, A47

Sánchez, S. F., Pérez, E., Sánchez-Blázquez, P., et al. 2016, Rev. Mex. Astron. Astrofis., 52, 21

Sánchez-Blázquez, P., Rosales-Ortega, F. F., Méndez-Abreu, J., et al. 2014, A\&A, 570, A6

Sandage, A. 1986, A\&A, 161, 89

Sanders, D. B., \& Mirabel, I. F. 1996, ARA\&A, 34, 749

Sarzi, M., Falcón-Barroso, J., Davies, R. L., et al. 2006, MNRAS, 366, 1151

Scalo, J. M., \& Struck-Marcell, C. 1986, ApJ, 301, 77

Schaerer, D., Charbonnel, C., Meynet, G., Maeder, A., \& Schaller, G. 1993, A\&AS, 102, 339

Schaller, G., Schaerer, D., Meynet, G., \& Maeder, A. 1992, A\&AS, 96, 269

Schiminovich, D., Wyder, T. K., Martin, D. C., et al. 2007, ApJS, 173, 315

Schmidt, M. 1959, ApJ, 129, 243

Schmidt, M. 1968, ApJ, 151, 393

Searle, L., Sargent, W. L. W., \& Bagnuolo, W. G. 1973, ApJ, 179, 427

Singh, R., van de Ven, G., Jahnke, K., et al. 2013, A\&A, 558, A43

Speagle, J. S., Steinhardt, C. L., Capak, P. L., \& Silverman, J. D. 2014, ApJS, 214,15

Stasińska, G., Vale Asari, N., Cid Fernandes, R., et al. 2008, MNRAS, 391, L29

Tacconi, L. J., Neri, R., Genzel, R., et al. 2013, ApJ, 768, 74

Tinsley, B. M. 1968, ApJ, 151, 547

Tinsley, B. M. 1972, A\&A, 20, 383

Tojeiro, R., Percival, W. J., Heavens, A. F., \& Jimenez, R. 2011, MNRAS, 413, 434

Vazdekis, A., Sánchez-Blázquez, P., Falcón-Barroso, J., et al. 2010, MNRAS, 404, 1639

Verheijen, M. A. W., Bershady, M. A., Andersen, D. R., et al. 2004, Astron. Nachr., 325, 151

Walcher, C. J., Wisotzki, L., Bekeraité, S., et al. 2014, A\&A, 569, A1

Walcher, J., Groves, B., Budavári, T., \& Dale, D. 2011, Ap\&SS, 331, 1

Wuyts, S., Förster Schreiber, N. M., van der Wel, A., et al. 2011, ApJ, 742, 96

Wuyts, S., Förster Schreiber, N. M., Nelson, E. J., et al. 2013, ApJ, 779, 135 


\section{Appendix A: Dependence of SFR on SSP models}

To evaluate to what extent our results depend on the choice of SSP models, we compare the properties derived with two bases: Base $G M e$, i.e., the one used in the main text and briefly described in Sect. 3.1, and base $C B e$, used in several earlier works by our group and fully described in González Delgado et al. (2015). In short, this base is built out of a preliminary update of the Bruzual \& Charlot (2003) models (Bruzual 2007, priv. comm.), from which we draw $N_{\star}=246$ elements with 41 ages (from 0.001 to $14 \mathrm{Gyr}$ ) and six metallicities $\left(\log Z / Z_{\odot}=-2.3\right.$, $-1.7,-0.7,-0.4,0$, and +0.4$)$. The evolutionary tracks are those collectively referred to as Padova (1994) by Bruzual \& Charlot (2003), and the IMF is that of Chabrier (2003). Compared to $G M e$, base $C B e$ differs in evolutionary tracks, IMF, and metallicity range.

We make two types of comparisons in this appendix: (i) global (galaxy wide) quantities, such as the current and initial stellar masses and the total SFR; and (ii) radial averages of $\mu_{\star}$, $A_{V}, \Sigma_{\mathrm{SFR}}, \Sigma_{\mathrm{SFR}} / \mu_{\star}$, and $x_{Y}$ for up to a maximum 30 of points for each galaxy (corresponding to $R=0-3$ in steps of $0.1 \mathrm{HLR}$ ). Figure A.1 shows the results with base GMe values in the $x$-axis and $C B e$ ones in the $y$-axis. Each panel shows a one-to-one line, as well as the mean $(\bar{\Delta})$ and standard deviation $(\sigma)$ of the difference $\Delta \equiv \operatorname{property}(\mathrm{CBe})-\operatorname{property}(\mathrm{GMe})$.

On average, $G M e$-based $M_{\star}$ and $\mu_{\star}$-values are $\sim 0.26 \mathrm{dex}$ higher than the corresponding $C B e$-based values, reflecting the different IMFs used. Discounting this offset, the two values of stellar mass and mass surface density agree to within 0.06 and 0.11 dex, respectively. In terms of the initial mass that is converted into stars $\left(M_{\text {ini }}\right)$, there is a difference of 0.12 dex between the two bases and a dispersion of 0.06 dex. Again, the difference reflects the change of IMF between the two bases. We note that $\Delta \log M_{\star}$ is higher than $\Delta \log M_{\text {ini }}$ because the returned fraction $\mathcal{R}$ also differs from one base to the other $(\mathcal{R}=0.28$ and 0.48 for $G M e$ and $C B e$, respectively).

Owing to the IMF difference, the SFR should be lower for $C B e$ than for $G M e$. This is in fact the result (Fig.A.1d), but the difference is only 0.07 dex, lower than what we would expect from the change in IMF. This implies that besides the IMF, there are differences in the SFH between $G M e$ and $C B e$ and/or in stellar extinction. The latter explanation does not hold, since $A_{V}$ is very similar in the two sets of models with an offset of only $\Delta=0.03 \mathrm{mag}$ and dispersion $\sigma=0.06 \mathrm{mag}$. However, we note that there is an important difference between the light fraction in populations younger than $32 \mathrm{Myr}$. On average, $x_{Y}$ is $0.18 \mathrm{dex}$ higher with $C B e$ than $G M e$. This explains why the SFR with $C B e$, although lower than with $G M e$, is not a full factor of $\sim 1.7$ lower, as expected by the change of IMF.

This change in SFH and, in particular, in $x_{Y}$ does not produce any significant effect in the radial distribution of the star formation rate intensity, $\Sigma_{\mathrm{SFR}}(R)$. The two sets of values are correlated well (Fig. A.1g), with a tiny difference of $\Delta=-0.05$ dex (lower in $C B e$ than in $G M e$ ) and a dispersion $\sigma=0.15$ dex. The offset of $\Sigma_{\mathrm{SFR}} / \mu_{\star}$ between the two bases is $\Delta=0.23$ dex, mainly reflecting the offset in $\mu_{\star}$ due to the IMF (Fig. A.1b).
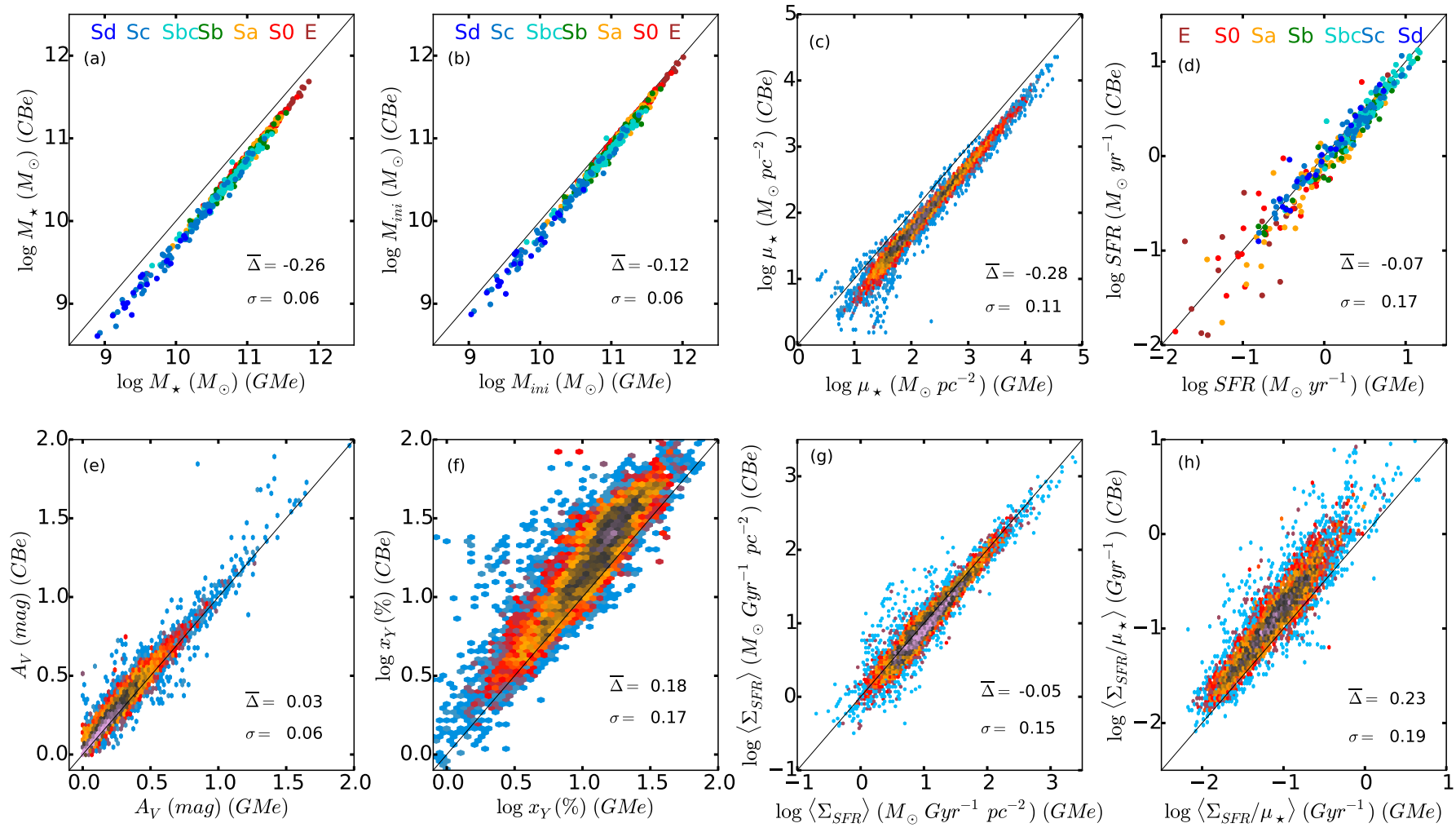

Fig. A.1. Comparison of several stellar population properties as obtained with the bases $G M e$ ( $x$-axis) and $C B e$ ( $y$-axis). The average difference between the property in the $y$ - and $x$-axis is labeled as $\bar{\Delta}$ in each panel, and its standard deviation as $\sigma$. Panels a), b), and d) show the galaxy mass and SFR, with galaxies colored by their Hubble type. In the other panels, the values of the property measured every 0.1 HLR are compared, and the color indicates the density of points in a logarithmic scale. 